\title{
Functional analysis on two-dimensional local fields
}

\author{
Alberto Cámara*
}

September 24, 2018

\begin{abstract}
We establish how a two-dimensional local field can be described as a locally convex space once an embedding of a local field into it has been fixed. We study the resulting spaces from a functional analytic point of view: in particular we study bounded, c-compact and compactoid submodules of two-dimensional local fields.
\end{abstract}

\section{Introduction}

This work is concerned with the study of characteristic zero two-dimensional local fields. These are complete discrete valuation fields whose residue field is a local field, either of characteristic zero or positive.

Following an idea introduced in [17, we do not regard two-dimensional fields as fields in the usual sense, but as an embedding of fields $K \hookrightarrow F$, where $K$ is a local field and $F$ is a two-dimensional local field. Given a two-dimensional local field $F$, such field embeddings always exist and we are not assuming any extra conditions on $F$; we are only changing our point of view.

In the arithmetic-geometric context, such field embeddings arise in the following way: suppose that $S$ is the spectrum of the ring of integers of a number field and that $f: X \rightarrow S$ is an arithmetic surface (for our purposes it is enough to suppose that $X$ is a regular 2 -dimensional scheme and that $f$ is projective and flat). Choose a closed point $x \in X$ and an irreducible curve $\overline{\{y\}} \subset X$ such that $x$ is regular in $\overline{\{y\}}$, and let $s=f(x) \in S$. Starting from the local ring of regular functions $\mathcal{O}_{X, x}$, we obtain a two-dimensional local field $F_{x, y}$ through a process of repeated completions and localizations:

$$
F_{x, y}=\operatorname{Frac}\left(\widehat{(\widehat{\mathcal{O} X, x})_{y}}\right) \text {. }
$$

This is analogue to the procedure of completion and localization that allows us to obtain a local field $K_{s}=\operatorname{Frac}\left(\widehat{\mathcal{O}_{S, s}}\right)$ from the closed point $s \in S$. The structure morphism $\mathcal{O}_{S, s} \rightarrow \mathcal{O}_{X, x}$ induces a field embedding $K_{s} \hookrightarrow F_{x, y}$.

The moral of the above paragraph is that if two-dimensional fields arise from an arithmetic-geometric context then they always come with a prefixed local field embedded into them.

\footnotetext{
*The author is supported by a Doctoral Training Grant at the University of Nottingham.
} 
What we study in this work is the $K$-vector space structure associated to $F$ via the embedding $K \hookrightarrow F$. As such, we connect the topological theory of twodimensional local fields with the theory of nonarchimedean locally convex vector spaces. In particular, for the fields $K((t))$ and $K\{\{t\}\}$ (see $\oint 2$ for the definition of the latter), we establish in Corollaries 3.2 and 3.8, a family of defining seminorms for the higher topology of the form

$$
\left\|\sum_{i} x_{i} t^{i}\right\|=\sup _{i}\left|x_{i}\right| q^{n_{i}},
$$

where $\left(n_{i}\right)_{i \in \mathbb{Z}} \subset \mathbb{Z} \cup\{-\infty\}$ is a sequence subject to certain conditions and $q$ is the number of elements in the residue field of $K$.

In particular, this provides us with a new way to describe higher topologies on two-dimensional local fields which does not rely on taking a lifting map from the residue field as in [15. This also introduces a new concept of bounded subset.

Although our description of higher topologies is valid for both equal and mixed characteristic two-dimensional local fields, the study of the functional theoretic properties in the two cases suggests that similarities stop here. Equal characteristic fields may be shown to be LF-spaces (see $\$ 1$ for the definition and Corollary 3.6) and, as such, they are bornological, nuclear and reflexive. This characterization is unavailable for mixed characteristic fields such as $K\{\{t\}\}$ and we show how these properties do not hold.

One of the advantages of our point of view is that certain submodules of $F$ arise as the families of c-compact and compactoid submodules, and therefore have a property which is a linear-topological analogue of compactness. In particular, compactoid submodules coincide with bounded submodules in equal characteristic (this is a consequence of nuclearity) and define a family strictly contained in that of bounded submodules in mixed characteristic. By using the associated bornology we achieve in Theorem 6.2 a very explicit self-duality result in the line of [4, Remark in $\S 3]$.

We briefly outline the contents and main results of this work. Sections $\$ 1$ and \$2 summarise relevant parts of the theory of nonarchimedean locally convex vector spaces and the structure of two-dimensional local fields, respectively. We have included them in this work in order to be able to refer to certain general results in later parts of the work and in order to fix notations and conventions. Hence, we do not supply proof for any statement in these sections, but refer the reader to the available literature.

At the beginning of section $\$ 3$ we center our attention in the $K$-vector spaces $K((t))$ and $K\{\{t\}\}$, two examples of two-dimensional local fields whose topological behaviour determines the topological properties of equal characteristic and mixed characteristic two-dimensional local fields, respectively. We prove in Propositions 3.1 and 3.7 how the higher topology on these two fields induces the structure of a locally convex $K$-vector space and we describe these locally convex topologies in terms of seminorms in Corollaries 3.2 and 3.8. We can easily study the case of $K((t))$, as in Proposition 3.4 we prove that the higher topology defines an LFspace and deduce the main analytic properties of this space in Corollary 3.6, it is complete, bornological, barrelled, reflexive and nuclear. In subsequent sections, we 
show how $K\{\{t\}\}$ is not bornological (Corollary 4.7), barrelled (Proposition 3.12), nuclear (Corollary 5.9) nor reflexive (Corollary 6.5).

Section $\$ 4$ deals with the nature of bounded subsets of the considered locally convex spaces. In particular, Propositions 4.2 and 4.4 describe a basis for the convex bornology associated to the locally convex topology, usually known as the VonNeumann bornology. We also prove in Proposition 4.9 that the multiplication map on the two-dimensional local field, despite not being continuous, is bounded.

$\$ 5$ contains a study of relevant $\mathcal{O}$-submodules of $K((t))$ and $K\{\{t\}\}$ such as rings of integers and rank-two rings of integers. In the case of $K((t))$, these may be shown to be c-compact (Proposition 5.3 and Corollary 5.4) but not compactoid (Corollary [5.5). In the case of $K\{\{t\}\}$, these rings of integers may be shown not to be compactoid nor c-compact (Corollary 5.11). It follows from nuclearity that all bounded submodules of $K((t))$ are compactoid. However, this is not the case for $K\{\{t\}\}$ : in Proposition 5.7 we describe a basis for the bornology of compactoid submodules on $K\{\{t\}\}$, which is strictly coarser than the Von-Neumann bornology. We are however able to prove that boundedness of the multiplication map on $K\{\{t\}\}$ holds for this coarser bornology (Corollary 5.12).

In $₫ 6$ we study duality issues. In particular, Theorem 6.2 establishes that the twodimensional local fields considered are isomorphic in the category of locally convex vector spaces to their appropriately topologized duals: we deduce some consequences of this fact. Finally, we study polarity issues after identifying our two-dimensional local fields and their duals.

In 37 we extend the results of the previous sections to the case of a general embedding $K \hookrightarrow F$ of a local field into a two-dimensional local field. It is important to remark that the functional analytic properties of equal characteristic two-dimensional local fields are the same as $K((t))$ and the properties of mixed characteristic two-dimensional local fields closely resemble those of $K\{\{t\}\}$.

Sections 98 and 99 explain how the results in this work can also be applied to archimedean two-dimensional local fields and positive characteristic local fields, respectively. In the first case, we are dealing with LF-spaces and we deduce our results from the well-established theory of (archimedean) locally convex spaces. In the second case, we relate the locally convex structure of vector spaces over $\mathbb{F}_{q}((u))$ to the linear topological structure of vector spaces over $\mathbb{F}_{q}$ through restriction of scalars. The study of two-dimensional local fields in positive characteristic using linear topological tools had been started by Parshin [19], and our point of view links with his in this case.

Finally, we discuss some applications and further directions of research in $\$ 10$,

Notation. Whenever $F$ is a complete discrete valuation field, we will denote by $\mathcal{O}_{F}, \mathfrak{p}_{F}, \pi_{F}$ and $\bar{F}$ its ring of integers, the unique nonzero prime ideal in the ring of integers, an element of valuation one and the residue field, respectively. Whenever $x \in \mathcal{O}_{F}, \bar{x} \in \bar{F}$ will denote its image modulo $\mathfrak{p}_{F}$. A two-dimensional local field is a complete discrete valuation field $F$ such that $\bar{F}$ is a local field.

Throughout the text, $K$ will denote a characteristic zero local field, that is, a finite extension of $\mathbb{Q}_{p}$ for some prime $p$. The cardinality of the finite field $\bar{K}$ will be denoted by $q$. The absolute value of $K$ will be denoted by $|\cdot|$, normalised so that $\left|\pi_{K}\right|=q^{-1}$. Due to far too many appearances in the text, we will ease notation by letting $\mathcal{O}:=\mathcal{O}_{K}, \mathfrak{p}:=\mathfrak{p}_{K}$ and $\pi:=\pi_{K}$. 
The conventions $\mathfrak{p}^{-\infty}=K, \mathfrak{p}^{\infty}=\{0\}$ and $q^{-\infty}=0$ will be used.

Acknowledgements. I am indebted to Matthew Morrow and Oliver Bräunling, with whom I had the initial discussions that later turned into this piece of work. Thomas Oliver has been my counterpart in many interesting conversations during the process of establishing and writing down the results contained here. I am also in great debt with Ralf Meyer and Cristina Pérez-García, whose comments on early drafts of this work have proven to be invaluable. I would also like to thank the anonymous referee, whose comments and criticism have led me to improve the text considerably. Finally, I thank my supervisor Ivan Fesenko for his guidance and encouragement.

\section{Locally convex spaces over $K$}

In this section we summarise some concepts and fix some notation regarding locally convex vector spaces over $K$. This is both for the reader's convenience as much as for establishing certain statements and properties for later reference.

The theory of locally convex vector spaces over a nonarchimedean field is well developed in the literature, so we will keep a concise exposition of the facts that we will require later. Both [21] and 20] are very good references on the topic.

Let $V$ be a $K$-vector space. A lattice in $V$ is an $\mathcal{O}$-submodule $\Lambda \subseteq V$ such that for any $v \in V$ there is an element $a \in K^{\times}$such that $a v \in \Lambda$. This is equivalent to having

$$
\Lambda \otimes_{\mathcal{O}} K \cong V
$$

as $K$-vector spaces. A subset of $V$ is said to be convex if it is of the form $v+\Lambda$ for $v \in V$ and $\Lambda$ a lattice in $V$. A vector space topology on $V$ is said to be locally convex if the filter of neighbourhoods of zero admits a collection of lattices as a basis.

A seminorm on $V$ is a map $\|\cdot\|: V \rightarrow \mathbb{R}$ such that:

(i) $\|\lambda v\|=|\lambda| \cdot\|v\|$ for every $\lambda \in K, v \in V$,

(ii) $\|v+w\| \leq \max (\|v\|,\|w\|)$ for all $v, w \in V$.

These conditions imply in particular that a seminorm only takes non-negative values and that $\|0\|=0$. A seminorm $\|\cdot\|$ is said to be a norm if $\|x\|=0$ implies $x=0$.

The gauge seminorm of a lattice $\Lambda \subseteq V$ is defined by the rule:

$$
\|\cdot\|_{\Lambda}: V \rightarrow \mathbb{R}, \quad v \mapsto \inf _{v \in a \Lambda}|a| .
$$

Given a family of seminorms $\left\{\|\cdot\|_{j}\right\}_{j \in J}$ on $V$, there is a unique coarsest vector space topology on $V$ making the maps $\|\cdot\|_{j}: V \rightarrow \mathbb{R}$ continuous for every $j \in J$. Such topology is locally convex: since the intersection of a finite number of lattices is a lattice, the closed balls

$$
B_{j}(\varepsilon)=\left\{v \in V ;\|v\|_{j} \leq \varepsilon\right\}, \quad \varepsilon \in \mathbb{R}_{>0}, j \in J
$$

supply a subbasis of neighbourhoods of zero consisting of open lattices. Note that the use of the adjective closed here is, as usual in this setting, an imitation of the 
analogous archimedean convention. Topologically, $B_{j}(\varepsilon)$ and $\left\{v \in V ;\|v\|_{j}<\varepsilon\right\}$ are both open and closed.

A locally convex topology can be described in terms of lattices or in terms of seminorms; passing from one point of view to the other is a simple matter of language.

A locally convex vector space $V$ is said to be normable if its topology may be defined by a single norm. By saying that $V$ is normed $K$-vector space, we will imply that we are considering a norm on it and that we regard the space together with the locally convex topology defined by a norm.

For a locally convex vector space $V$, a subset $B \subset V$ is bounded if for any open lattice $\Lambda \subset V$ there is an $a \in K$ such that $B \subseteq a \Lambda$. Alternatively, $B$ is bounded if for every continuous seminorm $\|\cdot\|$ on $V$ we have

$$
\sup _{v \in B}\|v\|<\infty .
$$

A locally convex $K$-vector space $V$ is bornological if any seminorm which is bounded on bounded sets is continuous. A linear map between locally convex vector spaces $V \rightarrow W$ is said to be bounded if the image of any bounded subset of $V$ is a bounded subset of $W$.

More generally, a bornology on a set $X$ is a collection $\mathcal{B}$ of subsets of $X$ which cover $X$, is hereditary under inclusion and stable under finite union. We say that the elements of $\mathcal{B}$ are bounded sets and the pair $(X, \mathcal{B})$ is referred to as a bornological space [10, Chapter I].

Just like a topology on a set is the minimum amount of information required in order to have a notion of open set and continuous map, a bornology on a set is the minimum amount of information required in order to have a notion of bounded set and bounded map, the latter being a map between two bornological spaces which preserves bounded sets. A basis for a bornology $\mathcal{B}$ on a set is a subfamily $\mathcal{B}_{0} \subset \mathcal{B}$ such that every element of $\mathcal{B}$ is contained in an element of $\mathcal{B}_{0}$.

The bornology which we have described above for a locally convex vector space $V$ is known as the Von-Neumann bornology [9, §I.2], and it is compatible with the vector space structure, meaning that the vector space operations are bounded maps. Moreover, the Von-Neumann bornology on a locally convex vector space is convex, as it admits a basis given by convex subsets [9, §I.6].

Open lattices in a non-archimedean locally convex space are also closed [21, textsection 6]. A locally convex space $V$ is said to be barrelled if any closed lattice is open.

Among many general ways to construct locally convex spaces [21, §5], we will require the use of products.

Proposition 1.1. Let $\left\{V_{i}\right\}_{i \in I}$ be a family of locally convex $K$-vector spaces, and let $V=\prod_{i \in I} V_{i}$. Then the product topology on $V$ is locally convex.

Proof. See [21, §5.C]. If $\left\{\Lambda_{i, j}\right\}_{j}$ denotes the set of open lattices of $V_{i}$ for $i \in I$, then the set of open lattices of $V$ is given by finite intersections of lattices of the form $\pi_{i}^{-1} \Lambda_{i, j}$. 
Equivalently, the product topology on $V$ is the one defined by all seminorms of the form

$$
v \mapsto \sup _{i, j}\left\|\pi_{i}(v)\right\|_{i, j},
$$

where $\left\{\|\cdot\|_{i, j}\right\}_{j}$ is a defining family of seminorms for $V_{i}$ for all $i \in I, \pi_{i}: V \rightarrow V_{i}$ is the corresponding projection and the supremum is taken over a finite collection of indices $i, j$.

Similarly, if $\left(X_{i}, \mathcal{B}_{i}\right)_{i \in I}$ is a collection of bornological sets, the product bornology on $X=\prod_{i \in I} X_{i}$ is the one defined by taking as a basis the sets of the form $B=$ $\prod_{i \in I} B_{i}$ with $B_{i} \in \mathcal{B}_{i}[10, \S 2.2]$.

Another construction which we will require is that of inductive limits. Let $V$ be a $K$-vector space and $\left\{V_{i}\right\}_{i \in I}$ be a collection of locally convex $K$-vector spaces. Let, for each $i \in I, f_{i}: V_{i} \rightarrow V$ be a $K$-linear map. The final topology for the collection $\left\{f_{i}\right\}_{i \in I}$ is not locally convex in general. However, there is a finest locally convex topology on $V$ making the map $f_{i}$ continuous for every $i \in I$ [21, §5.D]. That topology is called the locally convex final topology on $V$. Inductive limits and direct sums of locally convex spaces are particular examples of such construction.

Definition 1.2. Suppose that $V$ is a $K$-vector space and that we have an increasing sequence of vector subspaces $V_{1} \subseteq V_{2} \subseteq \cdots \subseteq V$ such that $V=\cup_{n \in \mathbb{N}} V_{n}$. Suppose that for each $n \in \mathbb{N}, V_{n}$ is equipped with a locally convex topology such that $V_{n} \hookrightarrow$ $V_{n+1}$ is continuous. Then the final locally convex topology on $V$ is called the strict inductive limit topology.

Let us fix, from now until the end of the present section, a locally convex vector space $V$. In order to discuss completeness issues, we require to deal not only with sequences, but arbitrary nets.

Let $I$ be a directed set. A net in $V$ is a family of vectors $\left(v_{i}\right)_{i \in I} \subset V$. A sequence is a net which is indexed by the set of natural numbers. The net $\left(v_{i}\right)_{i \in I}$ converges to a vector $v$, and we shall write $v_{i} \rightarrow v$, if for any $\varepsilon>0$ and continuous seminorm $\|\cdot\|$ on $V$, there is an index $i \in I$ such that for every $j \geq i$ we have $\left\|v_{j}-v\right\| \leq \varepsilon$. Similarly, the net $\left(v_{i}\right)_{i \in I}$ is said to be Cauchy if for any $\varepsilon>0$ and continuous seminorm $\|\cdot\|$ on $V$ there is an index $i \in I$ such that for every pair of indices $j, k \geq i$ we have $\left\|v_{j}-v_{k}\right\| \leq \varepsilon$.

Definition 1.3. A subset $A \subseteq V$ is said to be complete if any Cauchy net in $A$ converges to a vector in $A$.

A $K$-Banach space is a complete normed locally convex vector space. $V$ is said to be a Fréchet space if it is complete and its locally convex topology is metrizable. A locally convex vector space is said to be an $L F$-space if it may be constructed as an inductive limit of a family of Fréchet spaces.

Example 1.4. $K$ is a Fréchet space. There is a unique locally convex topology on any finite dimensional $K$-vector space which defines a structure of Fréchet space [21, Proposition 4.13]. 
In general, the usual topological notion of compactness is not very powerful for the study of infinite dimensional vector spaces over non-archimedean fields. This is why we prefer to use the language of c-compactness, which is an $\mathcal{O}$-linear concept of compactness.

Definition 1.5. Let $A$ be an $\mathcal{O}$-submodule of $V$. $A$ is said to be $c$-compact if, for any decreasing filtered family $\left\{\Lambda_{i}\right\}_{i \in I}$ of open lattices of $V$, the canonical map

$$
A \rightarrow \lim _{i \in I} A /\left(\Lambda_{i} \cap A\right)
$$

is surjective.

Example 1.6. The base field $K$ is c-compact as a $K$-vector space [21, §12]. This shows that a c-compact module need not be bounded.

This property may be phrased in a more topological way.

Proposition 1.7. An $\mathcal{O}$-submodule $A \subseteq V$ is c-compact if and only if for any family $\left\{C_{i}\right\}_{i \in I}$ of closed convex subsets $C_{i} \subseteq A$ such that $\bigcap_{i \in I} C_{i}=\emptyset$ there are finitely many indices $i_{1}, \ldots, i_{m} \in I$ such that $C_{i_{1}} \cap \ldots \cap C_{i_{m}}=\emptyset$.

Proof. See [21, Lemma 12.1.ii and subsequent paragraph].

Proposition 1.8. Let $\left\{V_{h}\right\}_{h \in H}$ be a collection of locally convex $K$-vector spaces, and for each $h \in H$ let $A_{h} \subseteq V_{h}$ be a c-compact $\mathcal{O}$-submodule. Then $\prod_{h \in H} A_{h}$ is c-compact in $\prod_{h \in H} V_{h}$.

Proof. [21, Prop. 12.2].

Another notion which is used in this setting is that of a compactoid $\mathcal{O}$-module; it is a notion which is analogous to that of relative compactness in the archimedean setting.

Definition 1.9. Let $A \subseteq V$ be an $\mathcal{O}$-submodule. $A$ is compactoid if for any open lattice $\Lambda$ of $V$ there are finitely many vectors $v_{1}, \ldots, v_{m} \in V$ such that

$$
A \subseteq \Lambda+\mathcal{O} v_{1}+\cdots+\mathcal{O} v_{m}
$$

Let $A \subseteq V$ be an $\mathcal{O}$-submodule. If $A$ is c-compact, then it is closed and complete. Similarly, if $A$ is compactoid then it is bounded. [21, §12].

Proposition 1.10. Let $A \subseteq V$ be an $\mathcal{O}$-submodule. The following are equivalent.

(i) A is c-compact and bounded.

(ii) A is compactoid and complete.

Proof. [21, Prop. 12.7].

The collection of compactoid $\mathcal{O}$-submodules of $V$ generates a bornology which is a priori weaker than the one given by the locally convex topology. 
Remark 1.11. It should be pointed out that the locally convex vector spaces that we consider in this work are always defined over a local field, which is discretely valued and, therefore, locally compact and spherically complete. This implies that the general theory of locally convex spaces over a nonarchimedean complete field simplifies in our setting. In particular, for an $\mathcal{O}$-submodule $A \subseteq V$, compactoidness and completeness imply compactness [20, Theorem 3.8.3]. We choose, however, to use the language of c-compact and compactoid submodules.

If $V, W$ are two locally convex $K$-vector spaces, a linear map $f: V \rightarrow W$ is continuous as soon as the pull-back of a continuous seminorm is a continuous seminorm. We denote the $K$-vector space of continuous linear maps between $V$ and $W$ by $\mathcal{L}(V, W)$.

The space $\mathcal{L}(V, W)$ may be topologized in the following way. Let $\mathcal{B}$ be a collection of bounded subsets of $V$. For any continuous seminorm $\|\cdot\|$ on $W$ and $B \in \mathcal{B}$, consider the seminorm

$$
\|\cdot\|_{B}: \mathcal{L}(V, W) \rightarrow \mathbb{R}, \quad f \mapsto \sup _{v \in B}\|f(v)\| .
$$

Definition 1.12. We write $\mathcal{L}_{\mathcal{B}}(V, W)$ for the space of continuous linear maps from $V$ to $W$ endowed with the locally convex topology defined by the seminorms $\|\cdot\|_{B}$, for every continuous seminorm $\|\cdot\|$ on $W$ and $B \in \mathcal{B}$.

In the particular case in which $\mathcal{B}$ consists of all bounded sets of $V$, we write $\mathcal{L}_{b}(V, W)$ for the resulting space, which is then said to have the topology of uniform convergence, or b-topology. If $\mathcal{B}$ consists only of the singletons $\{v\}$ for $v \in V$, we denote the resulting space by $\mathcal{L}_{s}(V, W)$ and say that it has the topology of point-wise convergence. Finally, if $\mathcal{B}$ is the collection of compactoid $\mathcal{O}$-submodules of $V$, we denote the resulting space by $\mathcal{L}_{c}(V, W)$ and say that it has the topology of uniform convergence on compactoid submodules, or c-topology.

There are two cases of particular interest: the topological dual space $V^{\prime}=$ $\mathcal{L}(V, K)$, and the endomorphism ring $\mathcal{L}(V)=\mathcal{L}(V, V)$. We denote $F_{s}^{\prime}, F_{b}^{\prime}, F_{c}^{\prime}, \mathcal{L}_{s}(V), \mathcal{L}_{b}(V)$ and $\mathcal{L}_{c}(V)$ for the corresponding topologies of point-wise convergence, uniform convergence and uniform convergence on compactoid submodules, respectively.

The choice of a family of bounded subsets $\mathcal{B}$ of $F$ does not affect $F_{\mathcal{B}}^{\prime}$ as a set, but it does affect the bidual space. As such, in the category of locally convex vector spaces over $K$, it is an interesting issue to classify which spaces are isomorphic, algebraically and/or topologically, to certain bidual spaces through the duality maps

$$
\delta: V \rightarrow\left(V_{\mathcal{B}}^{\prime}\right)^{\prime}, \quad v \mapsto \delta_{v}(l)=l(v) .
$$

The best possible case is when $\delta$ induces a topological isomorphism between $V$ and $\left(V_{b}^{\prime}\right)_{b}^{\prime}$; in this case we say that $V$ is reflexive.

Proposition 1.13. Every locally convex reflexive $K$-vector space is barrelled.

Proof. [21, Lemma 15.4].

The notion of polarity plays a role in the study of duality, as it provides us with a way of relating $\mathcal{O}$-submodules of $V$ to $\mathcal{O}$-submodules of $V^{\prime}$. 
Definition 1.14. If $A \subseteq V$ is an $\mathcal{O}$-submodule, we define its pseudo-polar by

$$
A^{p}=\left\{l \in V^{\prime} ;|l(v)|<1 \text { for all } v \in A\right\} .
$$

The pseudo-bipolar of $A$ is

$$
A^{p p}=\left\{v \in V ;|l(v)|<1 \text { for all } l \in A^{p}\right\} .
$$

Taking the pseudo-polar of an $\mathcal{O}$-submodule of $V$ gives an $\mathcal{O}$-submodule of $V^{\prime}$.

We have that $l \in A^{p}$ if and only if $l(A) \subseteq \mathfrak{p}$. Note that the traditional notion of polar relaxes the condition in the definition of pseudo-polar to $|l(v)| \leq 1$ or, equivalently, $l(A) \subseteq \mathcal{O}$. Introducing the distinction is an important technical detail, as pseudo-polarity is a better-behaved notion in the nonarchimedean setting.

Proposition 1.15. Let $A \subseteq V$ be an $\mathcal{O}$-submodule. We have

(i) If $A \subseteq B \subseteq V$ is another $\mathcal{O}$-submodule, then $B^{p} \subseteq A^{p}$.

(ii) $A^{p}$ is closed in $V_{s}^{\prime}$.

(iii) Let $\mathcal{B}$ be any collection of bounded subsets of $V$. If $A \in \mathcal{B}$, then $A^{p}$ is an open lattice in $V_{\mathcal{B}}^{\prime}$.

(iv) $A^{p p}$ is equal to the closure of $A$ in $V$.

Proof. Statements (i), (ii) and (iii) are part of [21, Lemma 13.1]. (iv) is [21, Proposition 13.4].

In order to conclude this section we define nuclear spaces. For any submodule $A \subseteq V$, denote $V_{A}:=A \otimes_{\mathcal{O}} K$, endowed with the locally convex topology associated to the gauge seminorm $\|\cdot\|_{A} \cdot V_{A}$ may not be a Hausdorff space, but its completion

$$
\widehat{V_{A}}:=\lim _{n \in \mathbb{Z}} V_{A} / \pi^{n} A
$$

is a $K$-Banach space.

Definition 1.16. $V$ is said to be nuclear if for any open lattice $\Lambda \subseteq V$ there exists another open lattice $M \subseteq \Lambda$ such that the canonical map $\widehat{V_{M}} \rightarrow \widehat{V_{\Lambda}}$ is compact, that is: there is an open lattice in $\widehat{V_{M}}$ such that the closure of its image is bounded and c-compact.

Proposition 1.17. We have:

(i) An $\mathcal{O}$-submodule of a nuclear space is bounded if and only if it is compactoid.

(ii) Arbitrary products of nuclear spaces are nuclear.

(iii) Strict inductive limits of nuclear spaces are nuclear.

Proof. (i) is [21, Proposition 19.2], (ii) is [21, Proposition 19.7] and (iii) is [21, Corollary 19.8]. 


\section{Our point of view on two-dimensional local fields}

We consider the category whose objects are field inclusions

$$
K \hookrightarrow F
$$

where $K$ is our fixed characteristic zero local field and $F$ is a two-dimensional local field. In such case, we shall say that $F$ is a two-dimensional local field over $K$. A morphism in this category between $K \hookrightarrow F_{1}$ and $K \hookrightarrow F_{2}$ is a commutative diagram of field inclusions

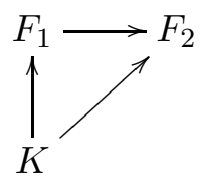

where $F_{1} \hookrightarrow F_{2}$ is an extension of complete discrete valuation fields.

The classification of characteristic zero two-dimensional local fields follows from Cohen structure theory of complete local rings and was established in [15]. The particular case with which we are dealing is very well described in [17, $\S 2.2$ and 2.3].

By this classification, given a two-dimensional local field $F$ it is always possible to exhibit a local field contained in it, so our assumption does not imply any further structure on $F$. Let us briefly recall the structure of two-dimensional local fields, which depends on the relation between the characteristics of $F$ and $\bar{F}$.

If char $F=\operatorname{char} \bar{F}$, the choice of a field embedding $\bar{F} \hookrightarrow F$ determines an isomorphism $F \cong \bar{F}((t))[8$, $\S I I .5]$. Such an isomorphism is not unique, as it does indeed depend on the choice of coefficient field $\bar{F} \hookrightarrow F$.

Besides fields of Laurent series, there is another construction which is key in order to work with two-dimensional local fields, and higher local fields in general. For any complete discrete valuation field $L$, consider

$$
L\{\{t\}\}=\left\{\sum_{i \in \mathbb{Z}} x_{i} t^{i} ; x_{i} \in L, \inf _{i \in \mathbb{Z}} v_{L}\left(x_{i}\right)>-\infty, x_{i} \rightarrow 0(i \rightarrow-\infty)\right\},
$$

with operations given by the usual addition and multiplication of power series. Note that we need to use convergence of series in $L$ in order to define the product. With the discrete valuation given by

$$
v_{L\{\{t\}\}}\left(\sum_{i \in \mathbb{Z}} x_{i} t^{i}\right):=\inf v_{L}\left(x_{i}\right),
$$

$L\{\{t\}\}$ turns into a complete discrete valuation field. In the particular case in which $L$ is a characteristic zero local field, the field $L\{\{t\}\}$ is a 2-dimensional local field which we call the standard mixed characteristic field over $L$. Its first residue field is $\bar{L}((\bar{t}))$.

We view elements of $L$ as elements of $L\{\{t\}\}$ in the obvious way. In particular, if $\pi_{L}$ is a uniformizer of $\mathcal{O}_{L}$, it is also a uniformizer of $\mathcal{O}_{L\{\{t\}\}}$; the element $t \in L\{\{t\}\}$ is such that $\bar{t} \in \bar{L}((\bar{t}))$ is a uniformizer.

Suppose now that $F$ is any two-dimensional local field such that $\operatorname{char} F \neq \operatorname{char} \bar{F}$. Then there is a unique field embedding $\mathbb{Q}_{p} \hookrightarrow F$. Let $\widetilde{K}$ be the algebraic closure of 
$\mathbb{Q}_{p}$ in $F$; it is a finite extension on $\mathbb{Q}_{p}$. In this case, $F$ contains a subfield which is $\widetilde{K}$-isomorphic to $\widetilde{K}\{\{t\}\}$, the extension $\widetilde{K}\{\{t\}\} \hookrightarrow F$ being finite. Finally, if a field embedding $K \hookrightarrow F$ with $K$ a local field is given, then we have $\mathbb{Q}_{p} \subseteq K \subseteq \widetilde{K}$ and the extension $K\{\{t\}\} \subseteq \widetilde{K}\{\{t\}\}$ is finite (details to all statements in this paragraph may be found in [17, §2.3.1]).

Since the residue field of a two-dimensional local field is a local field, we can use the discrete valuation at the residue level to define a rank-two valuation on $F$ as follows: order $\mathbb{Z} \oplus \mathbb{Z}$ by $\left(n_{1}, m_{1}\right)<\left(n_{2}, m_{2}\right)$ if and only if $n_{1}<n_{2}$ or $n_{1}=n_{2}$ and $m_{1}<m_{2}$ and consider, after choosing a uniformizer $\pi \in F$,

$$
\left(v_{F}, v_{\pi}\right): F^{\times} \rightarrow \mathbb{Z} \oplus \mathbb{Z}
$$

with $v_{\pi}(x):=v_{\bar{F}}\left(\overline{x \pi^{-v_{F}(x)}}\right)$. The valuation ring

$$
\mathrm{O}_{F}=\left\{x \in F ;\left(v_{F}(x), v_{\pi}(x)\right) \geq(0,0)\right\}
$$

does not depend on the choice of uniformizer [6, $\S 1]$.

Example 2.1. Consider $K=\mathbb{Q}_{p} \subset \mathbb{Q}_{p}\{\{t\}\}=F$. For the choice of uniformizer $p$ for $v_{F}$, the associated rank-two valuation of $F$ is

$$
\left(v_{1}, v_{2}\right): F^{\times} \rightarrow \mathbb{Z} \oplus \mathbb{Z}, \quad x=\sum_{i \in \mathbb{Z}} x_{i} t^{i} \mapsto\left(\inf _{i \in \mathbb{Z}} v_{p}\left(x_{i}\right), \inf \left\{i ; x_{i} \notin p^{v_{1}(x)+1} \mathbb{Z}_{p}\right\}\right) .
$$

The restriction of $v_{1}$ to $K$ is $v_{p}$, while $v_{2}$ restricts trivially. The rank-two ring of integers is

$$
\mathrm{O}_{F}=\left\{\sum_{i \in \mathbb{Z}} x_{i} t^{i} \in F ; x_{i} \in p \mathbb{Z}_{p} \text { for } i<0 \text { and } x_{i} \in \mathbb{Z}_{p} \text { for } i \geq 0\right\} .
$$

Example 2.2. Consider $K=\mathbb{Q}_{p} \subset \mathbb{Q}_{p}((t))=F$. In such case, the rank-two valuation of $F$ associated to the uniformizer $t$ for $v_{F}$ is

$$
\left(v_{1}, v_{2}\right): F^{\times} \rightarrow \mathbb{Z} \oplus \mathbb{Z}, \quad \sum_{i \geq i_{0}} a_{i} t^{i} \mapsto\left(i_{0}, v_{p}\left(a_{i_{0}}\right)\right),
$$

where we suppose that $a_{i_{0}}$ is the first nonzero coefficient in the power series. The restriction of $v_{1}$ to $K$ is trivial while the restriction of $v_{2}$ to $K$ is $v_{p}$. In this case we have $\mathrm{O}_{F}=\mathbb{Z}_{p}+t \mathbb{Q}_{p} \llbracket t \rrbracket$.

Remark 2.3. There are two particular local fields which play a very distinguished role when these objects are to be studied from a functional analytic point of view. Those are the fields $K((t))$ and $K\{\{t\}\}$. As we will see, most topological properties which hold in these particular cases will hold in general after taking restrictions of scalars or a base change over a finite extension which topologically is equivalent to taking a finite cartesian product. It is for this reason that we will work from now on with these two particular examples of two-dimensional local fields. We will explain how our results extend to the general case in $\$ 7$. 
Notation. When working with the two-dimensional local fields $F=K\{\{t\}\}$ or $F=K((t))$, for any collection $\left\{A_{i}\right\}_{i \in \mathbb{Z}}$ of subsets of $K$, we will denote

$$
\sum_{i \in \mathbb{Z}} A_{i} t^{i}=\left\{\sum_{i} x_{i} t^{i} \in F ; x_{i} \in A_{i} \text { for all } i \in \mathbb{Z}\right\} .
$$

We will also denote $\mathcal{O}_{K\{\{t\}\}}=\mathcal{O}\{\{t\}\}$. After all, this ring consists of all power series in $K\{\{t\}\}$ all of whose coefficients lie in $\mathcal{O}$.

\section{$3 \quad$ Higher topologies are locally convex}

In this section we will explain how the higher topology on $K((t))$ and $K\{\{t\}\}$ is a locally convex topology. Higher topologies for two-dimensional local fields were first introduced in [19] in the study of two-dimensional class field theory in positive characteristics. The general construction is available at [15], while [6, §1] contains an accessible survey on the topic.

We are forced to study both cases separately.

\subsection{Equal characteristic}

The higher topology on $K((t))$ is defined as follows. Let $\left\{U_{i}\right\}_{i \in \mathbb{Z}}$ be a collection of open neighbourhoods of zero in $K$ such that, if $i$ is large enough, $U_{i}=K$. Then define

$$
\mathcal{U}=\sum_{i \in \mathbb{Z}} U_{i} t^{i}
$$

The collection of sets of the form $\mathcal{U}$ defines the set of neighbourhoods of zero of a group topology on $K((t))[15, \S 1]$.

Proposition 3.1. The higher topology on $K((t))$ defines the structure of a locally convex $K$-vector space.

Proof. As $K$ is a local field, the collection of open neighbourhoods of zero admits a collection of open subgroups as a filter, that is: the basis of neighbourhoods of zero for the topology is generated by the sets of the form

$$
\mathfrak{p}^{n}=\left\{a \in K ; v_{K}(a) \geq n\right\},
$$

where $n \in \mathbb{Z} \cup\{-\infty\}$. These closed balls are not only subgroups, but $\mathcal{O}$-fractional ideals. This in particular means that the sets of the form

$$
\Lambda=\sum_{i \in \mathbb{Z}} \mathfrak{p}^{n_{i}} t^{i} \subseteq K((t))
$$

where $n_{i}=-\infty$ for large enough $i$, generate the higher topology. Moreover, they are not only additive subgroups, but also $\mathcal{O}$-modules.

If $x=\sum_{i \geq i_{0}} x_{i} t^{i} \in K((t))$ is an arbitrary element, and $i_{1}$ is such that $n_{i}=-\infty$ for all $i>i_{1}$ then we have the possibilities:

(i) $i_{0}>i_{1}$, in which case $x \in \Lambda$. 
(ii) $i_{0} \leq i_{1}$. In such case, let

$$
n=\max \left(\max _{i_{0} \leq i \leq i_{1}} n_{i}, 0\right) .
$$

Then $\pi^{n} \in \mathcal{O}$ satisfies $\pi^{n} x \in \Lambda$.

Thus, $\Lambda$ is a lattice and the higher topology is locally convex.

As a consequence of the previous proposition, it is possible to describe the higher topology in terms of seminorms.

Corollary 3.2. For any sequence $\left(n_{i}\right)_{i \in \mathbb{Z}} \subset \mathbb{Z} \cup\{-\infty\}$ such that there is an integer $k$ satisfying $n_{i}=-\infty$ for all $i>k$, define

$$
\|\cdot\|: K((t)) \rightarrow \mathbb{R}, \quad \sum_{i \gg-\infty} x_{i} t^{i} \mapsto \max _{i \leq k}\left|x_{i}\right| q^{n_{i}}
$$

Then $\|\cdot\|$ is a seminorm on $K((t))$ and the higher topology on $K((t))$ is the locally convex topology defined by the family of seminorms given by (5) as $\left(n_{i}\right)_{i \in \mathbb{Z}}$ varies over all sequences specified above.

Proof. This result is a consequence of Proposition 3.1 and of the fact that the gauge seminorm attached to a lattice of the form

$$
\Lambda=\sum_{i \in \mathbb{Z}} \mathfrak{p}^{n_{i}} t^{i}
$$

with $n_{i}=\infty$ for all $i>k$ is precisely the one given by (5). In order to see that, let $x=\sum_{i \geq i_{0}} x_{i} t^{i} \in K((t))$ and $a \in K$. We have that $x \in a \Lambda$ if and only if $x_{i} \in a \mathfrak{p}^{n_{i}}$ for every $i \geq i_{0}$. This is the case if and only if we have

$$
\left|x_{i}\right| q^{n_{i}} \leq|a|
$$

for all $i \geq i_{0}$. The infimum value of $|a|$ for which the above inequality holds is precisely the supremum of the values of $|x| q^{n_{i}}$ for $i \geq i_{0}$.

The seminorm $\|\cdot\|$ from the previous corollary is associated to and does depend on the choice of the sequence $\left(n_{i}\right)_{i \in \mathbb{Z}}$. If we have chosen notation not to reflect this fact, it is in hope that a lighter notation will simplify reading and that the sequence of integers defining $\|\cdot\|$, when needed, will be clear from the context.

Remark 3.3. As $F$ is a field, it is worth asking ourselves whether the seminorm (5) is multiplicative. It is very easy to check that for $i, j \in \mathbb{Z}$,

$$
\left\|t^{i}\right\| \cdot\left\|t^{j}\right\|=q^{n_{i}+n_{j}}
$$

while

$$
\left\|t^{i+j}\right\|=q^{n_{i+j}}
$$

These two values need not coincide in general. 
The field of Laurent series $K((t))$ has been considered previously from the point of view of the theory of locally convex spaces in the following manner. The ring of Taylor series $K \llbracket t \rrbracket$ is isomorphic to $K^{\mathbb{N}}$ as a $K$-vector space, and thus might be equipped with the product topology of countably many copies of $K$. Moreover, we have

$$
K((t))=\cup_{i \in \mathbb{Z}} t^{i} K \llbracket t \rrbracket,
$$

with $t^{i} K \llbracket t \rrbracket \cong K^{\mathbb{N}}$. Therefore, we may topologize $K((t))$ as a strict inductive limit.

In the result below, we explain how the higher topology on $K((t))$ agrees with this description. We will immediately deduce most of the analytic properties of $K((t))$ from this result.

Proposition 3.4. The higher topology on $K((t))$ agrees with the strict inductive limit topology given by (6).

Proof. The open lattices for the product topology on $K^{\mathbb{N}}$ are exactly the ones of the form

$$
\prod_{i \in I} \Lambda_{i} \times \prod_{i \notin I} K
$$

where $I$ is a finite subset of $\mathbb{N}$ and $\Lambda_{i}$ are open lattices in $K$, that is, integer powers of $\mathfrak{p}$. This description agrees with the description of the open lattices in $K \llbracket t \rrbracket$ for the subspace topology induced by the higher topology.

Further, if $\Lambda=\sum_{i \in \mathbb{Z}} \mathfrak{p}^{n_{i}} t^{i}$ is an open lattice for the higher topology on $K((t))$, for any $j \in \mathbb{Z}$, we have that $\Lambda \cap t^{j} K \llbracket t \rrbracket=\sum_{i \geq j} \mathfrak{p}^{n_{i}} t^{i}$ is an open lattice for the product topology on $t^{j} K \llbracket t \rrbracket \cong K^{\mathbb{N}}$.

Finally, any open lattice for the strict inductive limit $\bigcup_{j \in \mathbb{Z}} t^{j} K \llbracket t \rrbracket$ is given by a collection of open lattices $\Lambda_{j} \subseteq t^{j} K \llbracket t \rrbracket$ for each $j \in \mathbb{Z}$. These are of the form $\Lambda_{j}=\sum_{i>j} \mathfrak{p}^{n_{i, j}} t^{i}$ for some sequence $\left(n_{i, j}\right)_{i \geq j} \subset \mathbb{Z} \cup\{-\infty\}$ for which there is an index $k_{i} \geq i$ such that $n_{i, j}=-\infty$ for all $j \geq k_{i}$. The fact that the inductive limit is strict amounts to the following: if $i_{1}<i_{2}$ then we have $n_{i_{1}, j}=n_{i_{2}, j}$ for every $j \geq i_{2}$ and, in particular, $k_{i_{1}}=k_{i_{2}}$. Altogether, this determines a sequence $\left(n_{i}\right)_{i \in \mathbb{Z}} \subset \mathbb{Z} \cup\{-\infty\}$ and an index $k \in \mathbb{Z}$ such that $n_{i}=-\infty$ for every $i \geq k$. Under the identification $K((t))=\bigcup_{j \in \mathbb{Z}} t^{j} K \llbracket t \rrbracket$, the lattice associated to $\left(\Lambda_{j}\right)_{j \in \mathbb{Z}}$ is $\Lambda=\sum_{i \in \mathbb{Z}} \mathfrak{p}^{n_{i}} t^{i}$, which is open for the higher topology.

Remark 3.5. The higher topology on $K((t))$ also admits the following description as an inductive limit. For each $i \in \mathbb{Z}$ and $j \geq i, t^{i} K[t] / t^{j} K[t]$ is a finite dimensional $K$-vector space and we endow it with its unique Hausdorff locally convex topology. The field of Laurent series might be constructed as

$$
K((t))=\underset{i \in \mathbb{Z}}{\lim } \underset{j \geq i}{\lim } t^{i} K[t] / t^{j} K[t]
$$

the higher topology on it agrees with the one obtained by endowing the direct and inverse limits in the above expression with the corresponding direct and inverse limit locally convex topologies. The proof of this statement is a restatement of Proposition 3.4.

Corollary 3.6. $K((t))$ is an LF-space. In particular, it is complete, bornological, barrelled, reflexive and nuclear. 
Proof. After Proposition 3.4, in order to see that $K((t))$ is an LF-space it suffices to check that the locally convex space $K^{\mathbb{N}}$ endowed with the product topology is a Fréchet space. This follows from the fact that $K$ itself is Fréchet and that a countable product of Fréchet spaces is a Fréchet space [20, Corollary 3.5.7].

Completeness follows from being a strict inductive limit of complete spaces [21, Lemma 7.9]. Being bornological and barrelled follow from [21, Proposition 8.2], reflexivity follows from [20, Corollary 7.4.23] and nuclearity follows from Proposition 1.17

\subsection{Mixed characteristic}

The higher topology on $K\{\{t\}\}$ may be described as follows.

Let $\left\{V_{i}\right\}_{i \in \mathbb{Z}}$ be a sequence of open neighbourhoods of zero in $K$ such that

(i) There is $c \in \mathbb{Z}$ such that $\mathfrak{p}^{c} \subseteq V_{i}$ for every $i \in \mathbb{Z}$.

(ii) For every $l \in \mathbb{Z}$ there is an index $i_{0} \in \mathbb{Z}$ such that $\mathfrak{p}^{l} \subseteq V_{i}$ for every $i \geq i_{0}$.

Then define

$$
\mathcal{V}=\sum_{i \in \mathbb{Z}} V_{i} t^{i} \subset K\{\{t\}\}
$$

The higher topology on $K\{\{t\}\}$ is the group topology defined by taking the sets of the form $\mathcal{V}$ as the collection of open neighbourhoods of zero [15, $\S 1]$.

Again, as $K$ is a local field, the collection of neighbourhoods of zero admits the collection of open subgroups as a filter. These are not only subgroups but $\mathcal{O}$ fractional ideals, namely the integer powers of the prime ideal $\mathfrak{p}$.

Proposition 3.7. Let $\left(n_{i}\right)_{i \in \mathbb{Z}} \subset \mathbb{Z} \cup\{-\infty\}$ be a sequence restricted to the conditions:

(i) There is $c \in \mathbb{Z}$ such that $n_{i} \leq c$ for every $i$.

(ii) For every $l \in \mathbb{Z}$ there is an index $i_{0} \in \mathbb{Z}$ such that $n_{i} \leq l$ for every $i \geq i_{0}$.

The set

$$
\Lambda=\sum_{i \in \mathbb{Z}} \mathfrak{p}^{n_{i}} t^{i}
$$

is an $\mathcal{O}$-lattice. The sets of the form (8) generate the higher topology on $K\{\{t\}\}$, which is locally convex.

Condition (ii) is equivalent, by definition of limit of a sequence, to having $n_{i} \rightarrow$ $-\infty$ as $i \rightarrow \infty$; we will phrase it this way in the future.

Proof. It is clear that $\Lambda$ is an $\mathcal{O}$-module, and that the conditions imposed on the indices $n_{i}$ imply that it is a basic neighbourhood of zero for the higher topology.

Given an arbitrary element $x=\sum_{i=-\infty}^{\infty} x_{i} t^{i} \in F$, we must show the existence of an element $a \in K^{\times}$such that $a x \in \Lambda$. Indeed, a power of the uniformizer does the trick: we have that $\pi^{n} x \in \Lambda$ if and only if $\pi^{n} x_{i} \in \mathfrak{p}^{n_{i}}$ for every $i \in \mathbb{Z}$, and this is true if and only if

$$
n+v_{K}\left(x_{i}\right) \geq n_{i}
$$


for all $i \in \mathbb{Z}$. In other words, such an $n$ exists if and only if the difference

$$
n_{i}-v_{K}\left(x_{i}\right)
$$

cannot be arbitrarily large. But on one hand there is an integer $c$ that bounds the $n_{i}$ from above, and on the other hand the values $v_{K}\left(x_{i}\right)$ are bounded below by $v_{F}(x)$. We may take $n=c-v_{F}(x)$.

Because the integer powers of $\mathfrak{p}$ generate the basis of neighbourhoods of zero of the topology on $K$, the lattices of the form (8) generate the higher topology. In particular, the higher topology on $K\{\{t\}\}$ is locally convex.

We wish to point out that condition (ii) for the sequence $\left(n_{i}\right)_{i \in \mathbb{Z}}$ has not been used in the proof. Indeed, such a condition may be suppressed and we would still obtain a locally convex topology on $K\{\{t\}\}$, if only finer: see Remark 4.8 for a description of the topology obtained in such case.

Once we know that the higher topology is locally convex, we can describe it in terms of seminorms.

Corollary 3.8. For any sequence $\left(n_{i}\right)_{i \in \mathbb{Z}} \subset \mathbb{Z} \cup\{-\infty\}$ satisfying the conditions:

(i) there is $c \in \mathbb{Z}$ such that $n_{i} \leq c$ for all $i \in \mathbb{Z}$,

(ii) $n_{i} \rightarrow-\infty$ as $i \rightarrow \infty$,

consider the seminorm

$$
\|\cdot\|: K\{\{t\}\} \rightarrow \mathbb{R}, \quad \sum_{i \in \mathbb{Z}} x_{i} t^{i} \mapsto \sup _{i \in \mathbb{Z}}\left|x_{i}\right| q^{n_{i}} .
$$

The higher topology on $K\{\{t\}\}$ is the locally convex topology generated by the family of seminorms defined by (9), as $\left(n_{i}\right)_{i \in \mathbb{Z}}$ varies over the sequences specified above.

Proof. The gauge seminorm associated to the lattice $\Lambda$ is (9). The argument is the same as the proof of Corollary 3.2 and we omit it.

The seminorms in Corollary 3.8 are well defined because they arise as gauge seminorms attached to lattices. If we forget this fact for a moment, let us examine the values $\left|x_{i}\right| q^{n_{i}}$.

On one hand, when $i$ tends to $-\infty$, the values $\left|x_{i}\right|$ tend to zero while the values $q^{n_{i}}$ stay bounded. On the other hand, when $i$ tends to $+\infty$ the values $\left|x_{i}\right|$ stay bounded and $q^{n_{i}}$ tends to zero. In conclusion, the values $\left|x_{i}\right| q^{n_{i}}$ are all positive and tend to zero when $|i| \rightarrow+\infty$; this implies the existence of their supremum.

Just like in the equal characteristic case, a defining seminorm $\|\cdot\|$ is not multiplicative, for the same reason.

A mixed characteristic two-dimensional local field cannot be viewed as a direct limit in a category of locally convex $K$-vector spaces in the fashion of Remark 3.5. However, such an approach is valid from an algebraic point of view in a category of $\mathcal{O}$-modules. 


\section{3 $\quad$ First topological properties}

For starters, let us recall a few well-known properties of higher topologies. A twodimensional local field $K \hookrightarrow F$ endowed with a higher topology is a Hausdorff topological group [15, Theorem 1.1.i and Proposition 1.2]. Moreover, multiplication by a fixed nonzero element defines a homeomorphism $F \rightarrow F$ [15, Theorem 1.1.ii] and the residue map $\mathcal{O}_{F} \rightarrow \bar{F}$ is open when $\mathcal{O}_{F}$ is given the subspace topology and the local field $\bar{F}$ is endowed with its usual complete discrete valuation topology $[3$, Proposition 3.6.(v)].

Remark 3.9. In order to show that $K((t))$ or $K\{\{t\}\}$ is Hausdorff, it suffices to show that given a nonzero element $x$, there is an admissible seminorm $\|\cdot\|$ for which $\|x\| \neq 0$. This is obvious.

Multiplication $\mu: F \times F \rightarrow F$ fails to be continuous when the product topology is considered on the left hand side [6, $\S 1.3 .2]$. However, $\mu$ is separately continuous as explained above.

Another well known fact about higher topologies is that no basis of open neighbourhoods of zero is countable [6, \$1.3.2]. In other words, these topologies do not satisfy the first countability axiom. This implies that the set of seminorms defining the higher topology is uncountable. From the point of view of functional analysis, this shows that two-dimensional local fields are not Fréchet spaces.

Definition 3.10. We will call seminorms of the form (5) in the equal characteristic case and (9) in the mixed characteristic case admissible.

In both cases, admissible seminorms are attached to a sequence $\left(n_{i}\right)_{i \in \mathbb{Z}} \subset \mathbb{Z} \cup$ $\{-\infty\}$, subject to different conditions, but satisfying the formula

$$
\left\|\sum_{i} x_{i} t^{i}\right\|=\sup _{i}\left|x_{i}\right| q^{n_{i}} ;
$$

the reasons why this formula is valid differ in each case.

Remark 3.11. Power series expressions of the form $x=\sum_{i} x_{i} t^{i}$ define convergent series with respect to the higher topology, in the sense that the net of partial sums $\left(\sum_{i \leq n} x_{i} t^{i}\right)_{n \in \mathbb{Z}}$ converges to $x$. If we let $S_{n}=\sum_{i \leq n} x_{i} t^{i}$ and $\|\cdot\|$ be any admissible seminorm, then

$$
\left\|x-S_{n}\right\|=\left\|\sum_{i>n} x_{i} t^{i}\right\|
$$

may be shown to be arbitrarily small if $n$ is large enough.

Another well-known fact is that rings of integers $K \llbracket t \rrbracket$ and $\mathcal{O}\{\{t\}\}$ are closed but not open. In the first case, consider the set of open (and closed) lattices

$$
\Lambda_{n}=\sum_{i \leq 0} \mathfrak{p}^{n} t^{i}+K \llbracket t \rrbracket, \quad n \geq 0
$$

to find that $K \llbracket t \rrbracket=\bigcap_{n \geq 0} \Lambda_{n}$ is closed. In the second case, consider the open (and closed) lattices:

$$
\Lambda_{n}=\sum_{i<n} K t^{i}+\mathcal{O} t^{n}+\sum_{i>n} K t^{i}, \quad n \in \mathbb{Z}
$$


and obtain that $\mathcal{O}\{\{t\}\}=\bigcap_{n \in \mathbb{Z}} \Lambda_{n}$ is closed. In order to see that these rings are not open, it is enough to say that they do not contain any open lattice.

A very similar argument shows that the rank-two rings of integers $\mathcal{O}+t K \llbracket t \rrbracket$ and $\sum_{i<0} \mathfrak{p} t^{i}+\sum_{i \geq 0} \mathcal{O} t^{i}$ are closed but not open.

After the previous remark, we get the following result.

Proposition 3.12. The field $K\{\{t\}\}$ is not barrelled.

Proof. The ring of integers $\mathcal{O}\{\{t\}\}$ is a lattice which is closed but not open.

\section{Bounded sets and bornology}

Let us describe the nature of bounded subsets of $K((t))$ and $K\{\{t\}\}$. We will supply a description of a basis for the Von-Neumann bornology of these fields.

Example 4.1. Let $\|\cdot\|$ be an admissible seminorm, attached to the sequence $\left(n_{i}\right)_{i \in \mathbb{Z}}$. The values of $\|\cdot\|$ on $\mathcal{O}$ only depend on $n_{0}$. If $n_{0}=-\infty$ then the restriction of $\|\cdot\|$ to $\mathcal{O}$ is identically zero. Otherwise, for any $x \in \mathcal{O}$ we have $\|x\| \leq q^{n_{0}}$ and therefore $\mathcal{O}$ is bounded.

Similarly, if $n_{0}>-\infty$, we may find elements $x \in K$ making the value $|x| q^{n_{0}}$ arbitrarily large. Hence, $K$ is unbounded.

Proposition 4.2. For any sequence $\left(k_{i}\right)_{i \in \mathbb{Z}} \subset \mathbb{Z} \cup\{\infty\}$ such that there is an index $i_{0} \in \mathbb{Z}$ for which $k_{i}=\infty$ for every $i<i_{0}$, consider the $\mathcal{O}$-submodule of $K((t))$ given by

$$
B=\sum_{i \in \mathbb{Z}} \mathfrak{p}^{k_{i}} t^{i}
$$

The bornology of $K((t))$ admits as a basis the collection of $\mathcal{O}$-submodules given by (10) as $\left(k_{i}\right)_{i \in \mathbb{Z}}$ varies over the sequences specified above.

Proof. First, the $\mathcal{O}$-submodule $B$ given by (10) is bounded: suppose that $\|\cdot\|$ is an admissible seminorm on $K((t))$ given by the sequence $\left(n_{i}\right)_{i \in \mathbb{Z}}$ and that $k$ is the index for which $n_{i}=-\infty$ for every $i>k$.

If $k<i_{0}$, then the restriction of $\|\cdot\|$ to $B$ is identically zero. Otherwise, for $x=\sum_{i \geq i_{0}} x_{i} t^{i} \in B$,

$$
\|x\|=\max _{i_{0} \leq i \leq k}\left|x_{i}\right| q^{n_{i}} \leq \max _{i_{0} \leq i \leq k} q^{n_{i}-k_{i}},
$$

and the bound is uniform for $x \in B$ once $\|\cdot\|$ has been fixed.

Next, we study general bounded sets. From Example 4.1 we deduce that if a subset of $K((t))$ contains elements for which one coefficient can be arbitrarily large, then the subset is unbounded in $F$. Therefore, any bounded subset of $K((t))$ is included in a subset of the form

$$
\sum_{i \in \mathbb{Z}} \mathfrak{p}^{k_{i}} t^{i}, \quad k_{i} \in \mathbb{Z} \cup\{\infty\}
$$

In order to prove our claim, it is enough to show that the indices $k_{i} \in \mathbb{Z} \cup\{\infty\}$ may be taken to be equal to $\infty$ for all small enough $i$.

We will show the contrapositive: a subset $D \subset K((t))$ cannot be bounded as soon as there is a decreasing sequence of indices $\left(i_{j}\right)_{j \geq 0} \in \mathbb{Z}_{<0}$ satisfying that for every 
$j \geq 0$ there is an element $\xi_{i_{j}} \in D$ with a nonzero coefficient in degree $i_{j}$, which we denote $x_{i_{j}} \in K$.

For, if such is the case, let

$$
n_{i}= \begin{cases}-\infty, & i \neq i_{j} \text { for any } j \geq 0 \text { or } i>0 \\ -i_{j}+v_{K}\left(x_{i_{j}}\right), & i=i_{j} \text { for some } j \geq 0\end{cases}
$$

and consider the associated admissible seminorm $\|\cdot\|$ on $K((t))$. We have

$$
\left\|\xi_{i_{j}}\right\| \geq\left|x_{i_{j}}\right| q^{n_{i_{j}}}=q^{-i_{j}}
$$

for every $j \geq 0$, and this shows that $D$ is not bounded.

Corollary 4.3. If $\|\cdot\|: K((t)) \rightarrow \mathbb{R}$ is a seminorm which is bounded on bounded sets, then there is an index $i_{0} \in \mathbb{Z}$ such that $\left\|t^{i}\right\|=0$ for all $i \geq i_{0}$.

Proof. Suppose that for every $i_{0} \in \mathbb{Z}$ there is an $i \geq i_{0}$ such that $\left\|t^{i}\right\| \neq 0$. If $i$ is such that $\left\|t^{i}\right\|>0$, take $k_{i} \in \mathbb{Z}$ such that

$$
q^{-k_{i}}\left\|t^{i}\right\| \geq q^{i} .
$$

If $i$ is such that $\left\|t^{i}\right\|=0$, take $k_{i}=0$. By Proposition 4.2, the set

$$
B=\sum_{i \geq 0} \mathfrak{p}^{k_{i}} t^{i}
$$

is bounded. Let $x_{j}=\pi_{K}^{k_{j}} t^{j}$ for every $j \geq 0$. We have that $\left\|x_{j}\right\|=q^{-k_{j}}\left\|t^{j}\right\|$. Our hypothesis implies that the sequence of real numbers $\left(\left\|x_{j}\right\|\right)_{j \geq 0}$ is unbounded, and therefore $\|\cdot\|$ is not bounded on $B$.

Proposition 4.4. Consider a sequence $\left(k_{i}\right)_{i \in \mathbb{Z}} \subset \mathbb{Z} \cup\{\infty\}$ which is bounded below. The bornology of $K\{\{t\}\}$ admits the $\mathcal{O}$-submodules of the form

$$
B=\sum_{i \in \mathbb{Z}} \mathfrak{p}^{k_{i}} t^{i}
$$

as a basis.

Proof. First, let us show that $B$ is bounded. Assume all the $k_{i}$ in (11) are bounded below by some integer $d$. Let $\|\cdot\|$ be an admissible seminorm on $K\{\{t\}\}$ defined by a sequence $\left(n_{i}\right)_{i \in \mathbb{Z}} \subset \mathbb{Z} \cup\{-\infty\}$. In particular, there is an integer $c$ such that $n_{i} \leq c$ for every $i \in \mathbb{Z}$.

Then, if $\sum x_{i} t^{i} \in B$, we have that

$$
\left\|\sum x_{i} t^{i}\right\|=\sup _{i}\left|x_{i}\right| q^{n_{i}} \leq q^{c-d},
$$

and the bound is uniform on $B$ once $\|\cdot\|$ has been fixed.

Next, we study general bounded sets. Again, from Example 4.1 we may deduce that a subset of $K\{\{t\}\}$ which contains elements with arbitrarily large coefficients 
cannot be bounded. Therefore, any bounded subset of $K\{\{t\}\}$ is contained in a set of the form

$$
\sum_{i \in \mathbb{Z}} \mathfrak{p}^{k_{i}} t^{i}, k_{i} \in \mathbb{Z} \cup\{\infty\} .
$$

In order to prove our claim, it is enough to show that the indices $k_{i}$ may be taken to be bounded below.

Suppose that $D \subset K\{\{t\}\}$ is not contained in a set of the form (11). Then it must contain elements with arbitrarily large coefficients. More precisely, at least one of the following must happen:

1. There is a decreasing sequence $\left(i_{j}\right)_{j \geq 0} \subset \mathbb{Z}_{<0}$ and a sequence $\left(\xi_{i_{j}}\right)_{j \geq 0} \subset D$ such that, if $x_{i_{j}} \in K$ denotes the coefficient in degree $i_{j}$ of $\xi_{i_{j}}$, we have $\left|x_{i_{j}}\right| \rightarrow \infty$ as $j \rightarrow \infty$.

2. There is an increasing sequence $\left(i_{j}\right)_{j \geq 0} \subset \mathbb{Z}_{\geq 0}$ and a sequence $\left(\xi_{i_{j}}\right)_{j \geq 0} \subset D$ such that, if $x_{i_{j}} \in K$ denotes the coefficient in degree $i_{j}$ of $\xi_{i_{j}}$, we have $\left|x_{i_{j}}\right| \rightarrow \infty$ as $j \rightarrow \infty$.

If condition 1 holds, consider the admissible seminorm $\|\cdot\|$ associated to the sequence

$$
n_{i}= \begin{cases}0, & \text { if } i \leq 0, \\ -\infty, & \text { if } i>0 .\end{cases}
$$

We have that $\left\|\xi_{i_{j}}\right\| \geq\left|x_{i_{j}}\right|$ for all $j \geq 0$ and this implies that $D$ cannot be bounded.

If condition 1 does not hold, then condition 2 must hold. In such case, define

$$
n_{i_{j}}= \begin{cases}\frac{v_{K}\left(x_{i_{j}}\right)-1}{2} & \text { if } v_{K}\left(x_{i_{j}}\right) \text { is odd } \\ \frac{v_{K}\left(x_{i_{j}}\right)}{2}, & \text { if } v_{K}\left(x_{i_{j}}\right) \text { is even. }\end{cases}
$$

Furthermore, let $n_{i}=-\infty$ for any $i<0$ and $n_{l}=n_{i_{j}}$ for any index $l$ such that $i_{j} \leq l<i_{j+1}$. With such choices, the following three facts hold:

(i) The sequence $\left(n_{i_{j}}-v_{K}\left(x_{i_{j}}\right)\right)_{j \geq 0}$ tends to infinity.

(ii) The sequence $\left(n_{i}\right)_{i \in \mathbb{Z}}$ is bounded above.

(iii) For any $l \in \mathbb{Z}$, there is an index $i_{0}$ such that $n_{i} \leq l$ for all $i \geq i_{0}$.

After (ii) and (iii), let $\|\cdot\|$ be the admissible seminorm associated to $\left(n_{i}\right)_{i \in \mathbb{Z}}$. We have, for every $j \geq 0,\left\|\xi_{i_{j}}\right\| \geq\left|x_{i_{j}}\right| q^{n_{i_{j}}}$, and thus $D$ cannot be bounded.

Definition 4.5. Given that they constitute a basis for the Von-Neumann topology, we will refer any $\mathcal{O}$-submodule of the form (10) (resp. (11)) as a basic bounded $\mathcal{O}$-submodule of $K((t))$ (resp. $K\{\{t\}\})$.

Corollary 4.6. If $\|\cdot\|: K\{\{t\}\} \rightarrow \mathbb{R}$ is a seminorm which is bounded on bounded sets, then there is a real number $C>0$ such that $\left\|t^{i}\right\|<C$ for every $i \in \mathbb{Z}$. 
Proof. Suppose that $\|\cdot\|$ is a seminorm such that the sequence of real numbers $\left(\left\|t^{i}\right\|\right)_{i \in \mathbb{Z}}$ is not bounded. Consider the bounded set

$$
\mathcal{O}\{\{t\}\}=\sum_{i \in \mathbb{Z}} \mathcal{O} t^{i}
$$

and the sequence $\left(t^{i}\right)_{i \in \mathbb{Z}} \subset \mathcal{O}\{\{t\}\}$. The seminorm $\|\cdot\|$ is not bounded on $\mathcal{O}_{F}$.

Contrary to the situation in the equal characteristic case, in the mixed characteristic setting we get the following.

Corollary 4.7. The space $K\{\{t\}\}$ is not bornological.

Proof. It is enough to supply a seminorm which is bounded on bounded sets but not continuous.

Consider the norm on $K\{\{t\}\}$ given by

$$
\left\|\sum_{i \in \mathbb{Z}} x_{i} t^{i}\right\|=\sup _{i \in \mathbb{Z}}\left|x_{i}\right|,
$$

which is the absolute value on $K\{\{t\}\}$ related to the valuation $v_{F}$. If $B$ is a basic bounded set as in (11), then

$$
\sup _{x \in B}\|x\|=\sup _{i \in \mathbb{Z}} q^{-k_{i}}
$$

and hence $\|\cdot\|$ is bounded on bounded sets. However, the norm $\|\cdot\|$ is not continuous on $K\{\{t\}\}$ because

$$
\mathcal{O}\{\{t\}\}=\{x \in K\{\{t\}\} ;\|x\| \leq 1\}
$$

is closed but not open in $K\{\{t\}\}$.

Remark 4.8. When defining the higher topology on $K\{\{t\}\}$, an admissible seminorm was attached to a sequence $\left(n_{i}\right)_{i \in \mathbb{Z}} \subset \mathbb{Z} \cup\{-\infty\}$ subject to two conditions:

(i) The $n_{i}$ are bounded above.

(ii) We have $n_{i} \rightarrow-\infty$ as $i \rightarrow \infty$.

However, in the proof of Proposition 3.7 we did not require to make use of condition (ii).

Indeed, if we remove condition (ii) and allow all sequences $\left(n_{i}\right)_{i \in \mathbb{Z}}$ satisfying only condition (i), we obtain a locally convex topology. Let us describe it: on one hand, the norm on $K\{\{t\}\}$ given by

$$
\sum_{i \in \mathbb{Z}} x_{i} t^{i} \mapsto \sup _{i \in \mathbb{Z}}\left|x_{i}\right|
$$

becomes continuous, as it corresponds to taking $n_{i}=0$ for all $i \in \mathbb{Z}$. Hence, the resulting locally convex topology is both finer than the higher topology and finer than the complete discrete valuation topology. It is an immediate exercise to see that under such a topology the ring of integers $\mathcal{O}\{\{t\}\}$ is a bounded open lattice and this is equivalent to the locally convex topology being defined by a single seminorm [21, 
Proposition 4.11]. We conclude that the resulting topology is the complete discrete valuation topology.

It is immediate to check that the complete discrete valuation topology on $K\{\{t\}\}$ defines a Banach $K$-algebra structure with very nice analytic properties. However, it is unclear whether this structure is of any arithmetic interest.

Proposition 4.9. Let $F=K((t))$ or $K\{\{t\}\}$. The multiplication map $\mu: F \times F \rightarrow F$ is bounded with respect to the product bornology on the domain.

Proof. Let $B_{1}=\sum_{i \in \mathbb{Z}} \mathfrak{p}^{m_{i}} t^{i}$ and $B_{2}=\sum_{i \in \mathbb{Z}} \mathfrak{p}^{n_{j}} t^{j}$ be two bounded $\mathcal{O}$-submodules of $F$. The product bornology on $F \times F$ is generated by sets of the form $B_{1} \times B_{2}$. We have that $\mu\left(B_{1}, B_{2}\right)=\sum_{k \in \mathbb{Z}} V_{k} t^{k}$ with $V_{k}=\sum_{k=i+j} \mathfrak{p}^{m_{i}} \mathfrak{p}^{n_{j}}=\sum_{k=i+j} \mathfrak{p}^{m_{i}+n_{j}}$. We distinguish cases.

If $F=K((t)), m_{i}=\infty$ and $n_{j}=\infty$ if $i$ and $j$ are small enough. In this case, the sum defining $V_{k}$ is actually finite and there is $l_{k} \in \mathbb{Z} \cup\{\infty\}$ such that $V_{k} \subset \mathfrak{p}^{l_{k}}$. Moreover, we actually have $V_{k}=\{0\}$ if $k$ is small enough and therefore $\mu\left(B_{1}, B_{2}\right) \subset F$ is bounded.

If $F=K\{\{t\}\}$, then there are integers $c$ and $d$ such that $m_{i} \geq c$ for all $i \in \mathbb{Z}$ and $n_{j} \geq d$ for all $j \in \mathbb{Z}$. This implies that $V_{k} \subset \mathfrak{p}^{c+d}$ for every $k$ and that it is bounded.

\section{Complete, c-compact and compactoid $\mathcal{O}$-submodules}

In this section we will study relevant $\mathcal{O}$-submodules of $K((t))$ and $K\{\{t\}\}$, including rings of integers and rank-2 rings of integers.

We start dealing with completeness of rings of integers.

Proposition 5.1. The rings of integers $K \llbracket t \rrbracket$ and $\mathcal{O}\{\{t\}\}$ are complete $\mathcal{O}$-submodules of $K((t))$ and $K\{\{t\}\}$, respectively.

In the case of $K \llbracket t \rrbracket \subset K((t))$, the result follows because $K((t))$ is complete and $K \llbracket t \rrbracket$ is a closed subset. However, it is also immediate to give an argument by hand.

Proof. Let $I$ be a directed set and $\left(x_{i}\right)_{i \in I}$ a Cauchy net in the ring of integers. We distinguish cases below.

In the case of $K \llbracket t \rrbracket$, we write $x_{i}=\sum_{k \geq 0} x_{k, i} t^{k}$ with $x_{k, i} \in K$. Since $\left(x_{i}\right)_{i \in I}$ is a Cauchy net in $\mathcal{O}_{F}$, we have that $\left(x_{k, i}\right)_{i \in I}$ is a Cauchy net in $K$ and hence converges to an element $x_{k} \in K$ for every $k \geq 0$. The element $x=\sum_{k \geq 0} x_{k} t^{k}$ is the limit of the Cauchy net.

In the case of $\mathcal{O}\{\{t\}\}$, the procedure is very similar. We write $x_{i}=\sum_{k \in \mathbb{Z}} x_{i, k} t^{k}$ with $x_{i, k} \in \mathcal{O}$. Since $\mathcal{O}$ is complete and $\left(x_{i, k}\right)_{i \in I}$ is a Cauchy net, it converges to an element $x_{k} \in \mathcal{O}$ for every $k \in \mathbb{Z}$. It is elementary to check that as $k \rightarrow-\infty$, we have $x_{k} \rightarrow 0$ and therefore $x=\sum_{k \in \mathbb{Z}} x_{k} t^{k}$ is a well-defined element in $\mathcal{O}\{\{t\}\}$ which is the limit of the Cauchy net.

Corollary 5.2. The rank-2 rings of integers of $K((t))$ and $K\{\{t\}\}$ are complete.

Proof. It follows from the previous proposition due to the fact that they are closed subsets of complete $\mathcal{O}$-submodules. 
Next we will study rings of integers from the point of view of c-compactness and compactoidness.

Proposition 5.3. $K \llbracket t \rrbracket i s$ c-compact.

Proof. As a locally convex $K$-vector space, $K \llbracket t \rrbracket$ is isomorphic to $K^{\mathbb{N}}$ (Proposition 3.4). The field $K$ is c-compact (Example 1.6). Finally, a product of c-compact spaces is c-compact (Proposition 1.8).

Corollary 5.4. The rank-2 ring of integers of $K((t)), \mathcal{O}+t K \llbracket t \rrbracket$, is c-compact.

Proof. After the previous proposition, the result follows from the fact that $\mathcal{O}+$ $t K \llbracket t \rrbracket \subset K \llbracket t \rrbracket$ is closed, as c-compactness is hereditary for closed subsets [21, Lemma 12.1.iii].

Corollary 5.5. The rings $K \llbracket t \rrbracket$ and $\mathcal{O}+t K \llbracket t \rrbracket$ are not compactoid.

Proof. This follows from the fact that they are both c-compact, unbounded, complete and Proposition 1.10.

The compactoid submodules of a locally convex vector space define a bornology. Since every compactoid submodule is bounded, in our case it is important to decide which basic bounded submodules of $K((t))$ and $K\{\{t\}\}$ are compactoid.

Since $K((t))$ is a nuclear space, the class of bounded $\mathcal{O}$-submodules and compactoid $\mathcal{O}$-submodules coincide [21, Proposition 19.2].

It is in any case easy to see that any basic bounded subset

$$
B=\sum_{i \geq i_{0}} \mathfrak{p}^{k_{i}} t^{i}, \quad k_{i} \in \mathbb{Z} \cup\{\infty\}
$$

is compactoid: suppose that $\Lambda=\sum_{i \in \mathbb{Z}} \mathfrak{p}^{n_{i}} t^{i}$ with $n_{i} \in \mathbb{Z} \cup\{-\infty\}$ and such that for every $i>i_{1}$ we have $n_{i}=-\infty$. If $i_{1}<i_{0}$ then $B \subset \Lambda$ and there is nothing to show. Otherwise, let $l_{i}=\min \left(n_{i}, k_{i}\right)$ for $i_{0} \leq i \leq i_{1}$. Then

$$
B \subseteq \Lambda+\sum_{i=i_{0}}^{i_{1}} \mathcal{O} \cdot \pi^{l_{i}} t^{i},
$$

which shows that it is compactoid.

Corollary 5.6. The basic bounded $\mathcal{O}$-submodules of $K((t))$ are $c$-compact.

Proof. In view of Proposition 1.10, it is enough to show that a submodule $B$ as in the proof of the previous proposition is complete for nets. But the argument for showing completeness of such $\mathcal{O}$-submodules is the same as in the proof of Proposition 5.1 and we shall omit it.

In the case of $K\{\{t\}\}$ there is a difference between bounded and compactoid $\mathcal{O}$-submodules. For the proof of the following proposition we will consider the projection maps

$$
\pi_{j}: K\{\{t\}\} \rightarrow K, \quad \sum_{i \in \mathbb{Z}} x_{i} t^{i} \mapsto x_{j}, \quad j \in \mathbb{Z} .
$$

These are examples of continuous nonzero linear forms on $K\{\{t\}\}$. 
Proposition 5.7. The only compactoid submodules amongst the basic bounded submodules of $K\{\{t\}\}$ are the ones of the form

$$
B=\sum_{i \in \mathbb{Z}} \mathfrak{p}^{k_{i}} t^{i}
$$

with $k_{i} \in \mathbb{Z}$ bounded below and such that $k_{i} \rightarrow \infty$ as $i \rightarrow-\infty$.

Proof. Let $B$ be a basic bounded submodule as in (13), with the $k_{i} \in \mathbb{Z} \cup\{\infty\}$ bounded below.

On one hand, assume that $k_{i} \rightarrow \infty$ as $i \rightarrow-\infty$. Let $\Lambda=\sum_{i \in \mathbb{Z}} \mathfrak{p}^{n_{i}} t^{i}$ be an open lattice and assume that $B$ is not contained in $\Lambda$, as otherwise there is nothing to prove. When $i \rightarrow \infty$, the $k_{i}$ are bounded below and $n_{i} \rightarrow-\infty$. Similarly, when $i \rightarrow-\infty, k_{i} \rightarrow \infty$ and the $n_{i}$ are bounded above. Hence, the following two statements are true:

(i) There is an index $i_{0}$ such that for every $i<i_{0}, k_{i} \geq n_{i}$.

(ii) There is an index $i_{1}$ such that for every $i>i_{1}, k_{i} \geq n_{i}$.

We have $i_{0} \leq i_{1}$, as otherwise $B$ is contained in $\Lambda$. Let $l_{i}=\min \left(k_{i}, n_{i}\right)$ for $i_{0} \leq i \leq i_{1}$. Then we have

$$
B \subseteq \Lambda+\sum_{i=i_{0}}^{i_{1}} \mathcal{O} \cdot \pi^{l_{i}} t^{i},
$$

which shows that $B$ is compactoid.

On the other hand, suppose that the $k_{i}$ do not tend to infinity as $i \rightarrow-\infty$. In such case, there is a decreasing sequence $\left(i_{j}\right)_{j \geq 0} \subset \mathbb{Z}_{<0}$ such that $\left(k_{i_{j}}\right)_{j \geq 0}$ is bounded above. Let $M \in \mathbb{Z}$ be such that $k_{i_{j}}<M$ for every $j \geq 0$.

Let

$$
\Lambda=\sum_{i<0} \mathfrak{p}^{M} t^{i}+\sum_{i \geq 0} K t^{i} \subset K\{\{t\}\}
$$

which is an open lattice. Suppose that $x_{1}, \ldots, x_{m} \in K\{\{t\}\}$ satisfy that $B \subseteq \Lambda+$ $\mathcal{O} x_{1}+\cdots+\mathcal{O} x_{m}$. We denote $x_{l}=\sum_{i \in \mathbb{Z}} x_{l, i} t^{i}$, with $x_{l, i} \in K$, for $1 \leq l \leq m$.

We know that for $1 \leq l \leq m$, we have $x_{l, i} \rightarrow 0$ as $i \rightarrow-\infty$. Therefore, there is an index $j_{0} \geq 0$ such that for every $j \geq j_{0}$ we have $v_{K}\left(x_{l, i_{j}}\right)>M$. Then we have

$$
\pi_{i_{j_{0}}}(B) \subseteq \pi_{i_{j_{0}}}\left(\Lambda+\mathcal{O} x_{1}+\cdots+\mathcal{O} x_{m}\right),
$$

from where we deduce

$$
\mathfrak{p}^{k_{j_{0}}} \subseteq \mathfrak{p}^{M}+\mathfrak{p}^{v_{K}\left(x_{1, i_{0}}\right)}+\cdots+\mathfrak{p}^{v_{K}\left(x_{m, i_{0}}\right)}=\mathfrak{p}^{M} .
$$

However, this inclusion contradicts the fact that $k_{i_{0}}<M$.

Definition 5.8. We will refer in the sequel to the $\mathcal{O}$-submodules of the form (10) (resp. (13)) as basic compactoid submodules of $K((t))$ (resp. $K\{\{t\}\})$.

We deduce several consequences of this result.

Corollary 5.9. The field $K\{\{t\}\}$ is not a nuclear space. 
Proof. After the previous Proposition and (i) in Proposition 1.17, the result follows by observing that $K\{\{t\}\}$ contains $\mathcal{O}$-submodules, such as $\mathcal{O}\{\{t\}\}$, which are bounded but not compactoid.

Corollary 5.10. The basic compactoid submodules of $K\{\{t\}\}$ are c-compact.

Proof. Again, in view of Proposition 1.10, it is enough to show that the $\mathcal{O}$-submodule $B$ as in (13) is complete. The argument is the same as in the proof of Proposition 5.1 and we omit it.

The proof of the following corollary is immediate after Proposition 5.7 .

Corollary 5.11. $\mathcal{O}\{\{t\}\}$ and the rank-two ring of integers of $K\{\{t\}\}$ are not compactoid nor c-compact.

Proof. The fact that these rings are not compactoid follows from Proposition 5.7 The fact that they are not c-compact follows from the fact that, on top of not being compactoid, they both are bounded and complete.

Corollary 5.12. Multiplication $\mu: K\{\{t\}\} \times K\{\{t\}\} \rightarrow K\{\{t\}\}$ is also bounded when $K\{\{t\}\}$ is endowed with the bornology generated by compactoid $\mathcal{O}$-submodules in the codomain, and the product of two copies of such bornology in the domain.

Proof. Let $B_{1}=\sum_{i \in \mathbb{Z}} \mathfrak{p}^{m_{i}} t^{i}$ and $B_{2}=\sum_{j \in \mathbb{Z}} \mathfrak{p}^{n_{j}} t^{j}$ be two basic compactoid $\mathcal{O}_{-}$ submodules of $K\{\{t\}\}$; let $V_{k}$ as in the proof of Proposition 4.9. Just like in the aforementioned proof, $V_{k}$ is a contained in a fractional ideal of $K$ and is therefore bounded. Moreover, it is possible to choose $l_{k} \in \mathbb{Z} \cup\{\infty\}$ such that $l_{k} \rightarrow \infty$ as $k \rightarrow-\infty$ and $V_{k} \subset \mathfrak{p}^{l_{k}}$; this proves that $\mu\left(B_{1}, B_{2}\right)$ is contained in a compactoid $\mathcal{O}$-submodule of $K\{\{t\}\}$.

\section{Duality}

Let us describe some duality issues of two-dimensional local fields when regarded as locally convex vector spaces over a local field.

Much is known about the self-duality of the additive group of a two-dimensional local field. From [4, §3], if $F$ is a two-dimensional local field, once a nontrivial continuous character

$$
\psi: F \rightarrow S^{1} \subset \mathbb{C}^{\times}
$$

has been fixed, the group of continuous characters of the additive group of $F$ consists entirely of characters of the form $\alpha \rightarrow \psi(a \alpha)$, where $a$ runs through all elements of $F$. This result is entirely analogous to the one-dimensional theory [22, Lemma 2.2.1].

In the case of $K((t))$ and $K\{\{t\}\}$, self-duality of the additive group follows in an explicit way from two self-dualities: that of the two-dimensional local field as a locally convex $K$-vector space, and that of the additive group of $K$ as a locally compact abelian group. Since the second is sufficiently documented [22, §2.2], let us focus on the first one.

We have already exhibited nontrivial continuous linear forms on a two-dimensional local field. Let $F=K((t))$ or $K\{\{t\}\}$; the map

$$
\pi_{i}: F \rightarrow K, \quad \sum x_{j} t^{j} \mapsto x_{i}
$$


is a continuous nonzero linear form for all $i \in \mathbb{Z}$.

Consider now the following map:

$$
\gamma: F \rightarrow F^{\prime}, \quad x \mapsto \pi_{x},
$$

with

$$
\pi_{x}: F \rightarrow K, \quad y \mapsto \pi_{0}(x y) .
$$

More explicitly, if $x=\sum x_{i} t^{i}$ and $y=\sum y_{i} t^{i}$, then

$$
\pi_{x}(y)=\sum x_{i} y_{-i}
$$

The map $\gamma$ is well-defined because $\pi_{x}$, being the composition of multiplication by a fixed element $F \rightarrow F$ and the projection $\pi_{0}: F \rightarrow K$, is a continuous linear form. Besides that, $\gamma$ is $K$-linear and injective.

Remark 6.1. Regarding topologies on dual spaces, we have that $K((t))_{c}^{\prime}=K((t))_{b}^{\prime}$ after Proposition 1.17,(i). However, the topology of $K\{\{t\}\}_{c}^{\prime}$ is strictly weaker than the one of $K\{\{t\}\}_{b}^{\prime}$ : consider the seminorm

$$
|\cdot| \mathcal{O}_{\{\{t\}\}}: K\{\{t\}\}^{\prime} \rightarrow \mathbb{R}, \quad l \mapsto \sup _{x \in \mathcal{O}\{\{t\}\}}|l(x)|,
$$

which is continuous with respect to the $b$-topology. If $|\cdot|_{\mathcal{O}\{\{t\}\}}$ was continuous with respect to the $c$-topology, there would be a basic compactoid submodule $B \subset K\{\{t\}\}$ and a constant $C>0$ such that $|l|_{\mathcal{O}\{\{t\}\}} \leq C|l|_{B}$ for all $l \in K\{\{t\}\}^{\prime}$.

However, suppose that $B=\sum_{i \in \mathbb{Z}} \mathfrak{p}^{k_{i}} t^{i}$ with $k_{i} \rightarrow \infty$ as $i \rightarrow-\infty$. For any real number $C>0$ there is an index $j \in \mathbb{Z}$ such that $C q^{-k_{j}}<1$. This implies the inequality

$$
C\left|\pi_{j}\right|_{B}<\left|\pi_{j}\right|_{\mathcal{O}\{\{t\}\}} .
$$

This shows that $|\cdot|_{\mathcal{O}\{\{t\}\}}$ is not continuous in the $c$-topology.

Theorem 6.2. The map $\gamma: F \rightarrow F_{c}^{\prime}$ is an isomorphism of locally convex $K$-vector spaces.

Before we prove this result, we need an auxiliary result.

Lemma 6.3. Let $w \in F^{\prime}$ and define, for every $i \in \mathbb{Z}, a_{i}=w\left(t^{-i}\right)$. Then the formal sum $\sum a_{i} t^{i}$ defines an element of $F$.

Proof. We distinguish cases. If $F=K((t))$, it is necessary to show that $a_{i}=0$ for all small enough indices $i$. In other words, that there is an index $i_{0} \in \mathbb{Z}$ such that for every $i \geq i_{0}$ we have $w\left(t^{i}\right)=0$. Without loss of generality, we may restrict ourselves to a continuous linear form $w: K \llbracket t \rrbracket \rightarrow K$. In this case we get our result from the following isomorphisms: first $K \llbracket t \rrbracket \cong K^{\mathbb{N}}$, second $\left(K^{\mathbb{N}}\right)^{\prime} \cong \bigoplus_{\mathbb{N}} K^{\prime}[20$, Theorem 7.4.22], and third $K^{\prime} \cong K$.

In the case in which $F=K\{\{t\}\}$, we need to show that the values $\left|a_{i}\right|$ for $i \in \mathbb{Z}$ are bounded and that $\left|a_{i}\right| \rightarrow 0$ as $i \rightarrow-\infty$. On one hand, the subset $\mathcal{O}\{\{t\}\} \subset F$ is bounded after Proposition 4.4 and $t^{i} \in \mathcal{O}\{\{t\}\}$ for every $i \in \mathbb{Z}$. As $w$ is continuous, the set $w(\mathcal{O}\{\{t\}\}) \subset K$ is bounded and therefore the values $w\left(t^{i}\right)$ are bounded. On the other hand, the net $\left(t^{i}\right)_{i \in \mathbb{Z}}$ tends to zero in $K\{\{t\}\}$ as $i \rightarrow \infty$. As $w$ is continuous, $a_{i}=w\left(t^{-i}\right) \rightarrow 0$ as $i \rightarrow-\infty$. 
Proof of Theorem 6.2. As explained above, the map $\gamma$ is well-defined, $K$-linear and injective.

Let $w \in F^{\prime}$. Define $x=\sum_{i} a_{i} t^{i} \in F$ with $a_{i}$ as in Lemma 6.3. Then, for $y=\sum y_{i} t^{i} \in F$, we have

$$
w\left(\sum y_{i} t^{i}\right)=\sum y_{i} w\left(t^{i}\right)=\sum y_{i} a_{-i}=\pi_{0}(x y)
$$

(the first equality follows from Remark 3.11). Therefore, $w=\pi_{x}$ and the map $\delta$ is surjective.

In order to show bicontinuity, let us first work out what continuity means in this setting. For any $\varepsilon>0$ and $B$ a set in the bornology generated by compactoid submodules, we must show that there are $\delta>0$ and an admissible seminorm $\|\cdot\|$ : $F \rightarrow K$ such that $\|x\| \leq \delta$ implies $\left|\pi_{x}\right|_{B} \leq \varepsilon$.

Without loss of generality, we may replace $\varepsilon$ and $\delta$ by integer powers of $q$, and the generic bounded set $B$ by a basic compactoid submodule of $F$, which is of the form (10) in the equal characteristic case or (13) in the mixed characteristic case. For convenience, let us write

$$
B=\sum_{i \in \mathbb{Z}} \mathfrak{p}^{k_{i}} t^{i}, \quad k_{i} \in \mathbb{Z} \cup\{\infty\}
$$

by allowing, in the equal characteristic case, $k_{i}=\infty$ for every small enough $i$.

Now, let $n \in \mathbb{Z}$. We take $n_{i}=-k_{-i}$ for every $i \in \mathbb{Z}$. Because of the conditions defining $B$, the sequence $\left(n_{i}\right)_{i \in \mathbb{Z}}$ defines an admissible seminorm $\|\cdot\|$ in both cases. Now, for $x=\sum x_{i} t^{i}$, we have that $\|x\| \leq q^{n}$ if and only if for every index $i \in \mathbb{Z}$ we have

$$
n_{i}-n \leq v_{K}\left(x_{i}\right) .
$$

Similarly, $\left|\pi_{x}\right|_{B} \leq q^{n}$ if and only if for every index $i \in \mathbb{Z}$ we have

$$
-k_{-i}-n \leq v_{K}\left(x_{i}\right)
$$

By direct comparison and substitution between (15) and (16), we have that with our choice of admissible seminorm $\|\cdot\|$,

$$
\|x\| \leq q^{n} \text { if and only if }\left|\pi_{x}\right|_{B} \leq q^{n},
$$

which shows bicontinuity.

Remark 6.4. In the mixed characteristic case we may ask ourselves if it is possible to exhibit any self-duality result involving $F_{b}^{\prime}$, that is, topologizing the dual space according to uniform convergence over all bounded sets.

It can be seen from the proof of Theorem 6.2 that this is not the case. Any bornology $\mathcal{B}$ stronger than the one generated by compactoid submodules will stop the map $\gamma: F \rightarrow F_{\mathcal{B}}^{\prime}$ from being continuous.

We remark that if there were no other bounded sets in $K\{\{t\}\}$ besides the ones generated by compactoid submodules, it would be possible to show that such a locally convex vector space is bornological.

From the failure of $K\{\{t\}\}$ at being bornological we may deduce that Theorem 6.2 is the best possible result. 
By applying Theorem 6.2 twice on $K((t))$, we recover the fact that this locally convex space is reflexive. Indeed, we have made this fact explicit via the choice of duality pairing:

$$
K((t)) \times K((t)) \rightarrow K, \quad(x, y) \mapsto \pi_{0}(x y) .
$$

Corollary 6.5. The field $K\{\{t\}\}$ is not reflexive.

Proof. Since any reflexive space is barrelled (Proposition 1.13), the result follows from Proposition 3.12

In order to conclude this section let us describe polars and pseudo-polars of the $\mathcal{O}$-submodules which we have studied in $\$ 5$.

After Theorem 6.2, the topological isomorphism given by $\gamma$ allows us to identify $F$ with $F_{c}^{\prime}$, and in particular lets us relate their $\mathcal{O}$-submodules.

Definition 6.6. Let $F=K((t))$ or $K\{\{t\}\}$. Let $A \subset F$ be an $\mathcal{O}$-submodule. We let

$$
A^{\gamma}=\gamma^{-1}\left(A^{p}\right) \subset F
$$

and refer to it, by abuse of language, as the pseudo-polar of $A$.

Proposition 6.7. Consider the $\mathcal{O}$-submodule

$$
A=\sum_{i \in \mathbb{Z}} \mathfrak{p}^{k_{i}} t^{i}, \quad k_{i} \in \mathbb{Z} \cup\{ \pm \infty\}
$$

of $F=K((t))$ or $K\{\{t\}\}$. Then, we have

$$
A^{\gamma}=\sum_{i \in \mathbb{Z}} \mathfrak{p}^{1-k_{-i}} t^{i}
$$

Proof. Let $B=\sum_{i \in \mathbb{Z}} \mathfrak{p}^{1-k_{-i}} t^{i}$.

On one hand, suppose $x=\sum x_{i} t^{i} \in B$. We have, for every $y=\sum y_{i} t^{i} \in A$,

$$
\left|\pi_{x}(y)\right|=\left|\sum x_{-i} y_{i}\right| \leq \sup \left|x_{-i}\right|\left|y_{i}\right|=\sup q^{-1+k_{i}-k_{i}}<1
$$

and, therefore, $B \subseteq A^{\gamma}$.

On the other hand, suppose that $x=\sum x_{i} t^{i} \in A^{\gamma}$. Then, by definition, we have

$$
\left|\pi_{x}(y)\right|<1, \quad \text { for any } y \in A .
$$

In particular, let $y=\pi^{k_{i}} t^{i}$. Then the inequality

$$
\left|\pi_{x}\left(\pi^{k_{i}} t^{i}\right)\right|=\left|x_{-i} \pi^{k_{i}}\right|<1
$$

implies that $v_{K}\left(x_{-i}\right) \geq 1-k_{i}$. Therefore $x_{-i} \in \mathfrak{p}^{1-k_{i}}$. Since our conclusion holds for any $i \in \mathbb{Z}$, we have that $x \in A^{\gamma}$ and, therefore, $B \subset A^{\gamma}$.

After (iv) in Proposition 1.15, we may think of the following corollary as a proof that the submodule $A$ in the statement of the previous Proposition is closed, as it is equal to its pseudo-bipolar; proofs to this result and the following two corollaries are immediate. 
Corollary 6.8. For an $\mathcal{O}$-submodule $A$ as in the previous Proposition, we have $A^{p p}=A$.

Corollary 6.9. We have $K \llbracket t \rrbracket^{\gamma}=K \llbracket t \rrbracket$. For the rank-2 ring of integers, we have $(\mathcal{O}+t K \llbracket t \rrbracket)^{\gamma}=\mathfrak{p}+t K \llbracket t \rrbracket$.

Corollary 6.10. We have $(\mathcal{O}\{\{t\}\})^{\gamma}=\mathfrak{p}\{\{t\}\}$. For the rank-2 ring of integers, we have $\left(\sum_{i<0} \mathfrak{p} t^{i}+\sum_{i \geq 0} \mathcal{O} t^{i}\right)^{\gamma}=\sum_{i \leq 0} \mathcal{O} t^{i}+\sum_{i>0} \mathfrak{p} t^{i}$.

By Proposition 1.15 and Theorem 6.2. pseudo-polarity exchanges open lattices and basic compactoid submodules. Under the characterization given by Proposition 6.7, the relation is evident.

The same arguments exposed apply to compute that the polar of the $\mathcal{O}$-submodule $\sum_{i \in \mathbb{Z}} \mathfrak{p}^{k_{i}} t^{i}, k_{i} \in \mathbb{Z} \cup\{ \pm \infty\}$ is $\sum_{i \in \mathbb{Z}} \mathfrak{p}^{-k_{-i}} t^{i}$. As such, the polar of an open lattice is a compactoid lattice and vice versa.

Let us write down a table with pseudo-polars and polars of relevant $\mathcal{O}$-submodules:

\begin{tabular}{l|l|l}
$\mathbf{A}$ & $\mathbf{A}^{\gamma}$ & polar of $\mathbf{A}$ \\
\hline$K \llbracket t \rrbracket$ & $K \llbracket t \rrbracket$ & $K \llbracket t \rrbracket$ \\
\hline $\mathcal{O}+t K \llbracket t \rrbracket$ & $\mathfrak{p}+t K \llbracket t \rrbracket$ & $\mathcal{O}+t K \llbracket t \rrbracket$ \\
\hline $\mathcal{O}\{\{t\}\}$ & $\mathfrak{p}\{\{t\}\}$ & $\mathcal{O}\{\{t\}\}$ \\
\hline$\sum_{i<0} \mathfrak{p} t^{i}+\sum_{i \geq 0} \mathcal{O} t^{i}$ & $\sum_{i \leq 0} \mathcal{O} t^{i}+\sum_{i>0} \mathfrak{p} t^{i}$ & $\sum_{i \leq 0} \mathcal{O} t^{i}+\sum_{i>0} \mathfrak{p}^{-1} t^{i}$ \\
\hline$\Lambda=\sum_{i \in \mathbb{Z}} \mathfrak{p}^{n_{i}} t^{i}$ & $B=\sum_{i \in \mathbb{Z}} \mathfrak{p}^{1-n_{-i}} t^{i}$ & $B=\sum_{i \in \mathbb{Z}} \mathfrak{p}^{-n_{-i}} t^{i}$ \\
(open lattice) & $($ compactoid) & $($ compactoid) \\
\hline$B=\sum_{i \in \mathbb{Z}} \mathfrak{p}^{k_{i}} t^{i}$ & $\Lambda=\sum_{i \in \mathbb{Z}} \mathfrak{p}^{1-k_{-i}} t^{i}$ & $B=\sum_{i \in \mathbb{Z}} \mathfrak{p}^{-k_{-i}} t^{i}$ \\
(basic compactoid) & $($ open lattice) & (open lattice)
\end{tabular}

The isomorphism $\gamma: F \rightarrow F_{c}^{\prime}$ is not unique, as it depends on choosing a nonzero linear form on $F$, which in our case is $\pi_{0}$. For example, replacing $\pi_{0}$ by $\pi_{1}$ in the definition of $\gamma$ would lead to an identical result. The actual shape of $A^{\gamma}$, for a given $\mathcal{O}$-submodule $A \subset F$, depends heavily on $\gamma$. However, the fact that polarity exchanges open lattices with compactoid submodules does not depend on $\gamma$.

In conclusion, taking the pseudo-polar or polar is a self-map on the set of $\mathcal{O}$ submodules of $K((t))$ or $K\{\{t\}\}$ which reverses inclusions, gives basic compactoid submodules when applied to open lattices and vice versa, and whose square equals the identity map when restricted to closed $\mathcal{O}$-submodules.

\section{General two-dimensional local fields}

In the previous sections of this work we have developed a systematic study of $K((t))$ and $K\{\{t\}\}$ from the point of view of the theory of locally convex spaces over $K$. Let us explain how the previous results extend to a general characteristic zero twodimensional local field $K \hookrightarrow F$. The moral of the story is that we can link the higher topology on $F$ to the constructions on $K((t))$ and $K\{\{t\}\}$ that we have performed in the preceding sections of this work by performing operations such as restriction 
of scalars along a finite extension and taking finite products, and due to their finite nature, none of these operations modifies the properties of the resulting locally convex spaces.

Due to the difference in their structures, we consider the equal characteristic and mixed characteristic cases separately.

\subsection{Equal characteristic}

Assume that $K \hookrightarrow F$ is a two-dimensional local field and that $\operatorname{char} F=\operatorname{char} \bar{F}$. In this case, as explained in 92 , the choice of a field embedding $\bar{F} \hookrightarrow F$ determines an isomorphism $F \cong \bar{F}((t))$.

Denote the algebraic closure of $K$ in $F$ by $\widetilde{K}$. The extension $\widetilde{K} \mid K$ is finite and $\widetilde{K} \hookrightarrow F$ is the only coefficient field of $F$ which factors the field inclusion $K \hookrightarrow F[17$, Lemma 2.7], and this is the only coefficient field of $F$ that we will take into account in our constructions.

Remark 7.1. It is a well-known fact that in this case the higher topology of $F$ depends on the choice of a coefficient field [23, Example 2.1.22]. This is why we stress that in this work the only coefficient field we consider is $\widetilde{K} \hookrightarrow F$ because the field embedding $K \hookrightarrow F$ is given a priori.

The $\widetilde{K}$-vector space $F \cong \widetilde{K}((t))$ is a complete, bornological, barrelled, reflexive and nuclear locally convex space by direct application of Corollary 3.6. The higher topology on $F$ only depends on the choice of the embedding $\widetilde{K} \hookrightarrow F$ and, therefore, does not change by restriction of scalars along $K \hookrightarrow \widetilde{K}$.

Let us explain this fact with more detail. On one hand, all open lattices $\Lambda$ are $\mathcal{O}_{\widetilde{K}}$-modules and hence also $\mathcal{O}$-modules by restriction of scalars. On the other hand, if $x \in F$, there is a positive power of $\pi_{\widetilde{K}}$ that maps $x$ to $\Lambda$ by multiplication. Uniformizers of $K$ and $\widetilde{K}$ may be chosen to be related by the ramification degree: $\pi=\pi_{\widetilde{K}}^{e(\widetilde{K} \mid K)}$. Therefore, there is a positive power of $\pi$ which maps $x$ to $\Lambda$ by multiplication and we deduce local convexity over $K$.

The absolute value on $\widetilde{K}$ restricts to the absolute value of $K$ and therefore Corollary 3.2 describes the admissible seminorms of $K \hookrightarrow F$ without any changes.

Moreover, after Proposition 3.4 we have that the higher topology on $\widetilde{K}((t))$ agrees with the strict inductive limit topology given by

$$
\widetilde{K}((t))=\bigcup_{i \in \mathbb{Z}} t^{i} \widetilde{K} \llbracket t \rrbracket
$$

which is also a union of $K$-vector spaces. Since the extension $\widetilde{K} \mid K$ is finite, we also have that $\widetilde{K} \llbracket t \rrbracket$ is isomorphic to a product of countably many copies of $K$ and is therefore a Fréchet $K$-vector space. Hence, we get that $F$ is an LF-space over $K$ and in particular we may deduce from Proposition 3.6 that $F$ is a complete, bornological, barrelled, reflexive and nuclear $K$-vector space.

Because admissible seminorms do not change after restricting scalars to $K$, Proposition 4.2 describes a basis of bounded $\mathcal{O}$-submodules of $F$. These are complete, and from nuclearity we deduce that the classes of bounded $\mathcal{O}$-submodules and compactoid $\mathcal{O}$-submodules of $F$ agree. 
Since $\widetilde{K}$ is a finite dimensional $K$-vector space, it is c-compact. The ring of integers $\mathcal{O}_{F}=\widetilde{K} \llbracket t \rrbracket$ is therefore c-compact, being isomorphic to a product of copies of $\widetilde{K}$. It is unbounded, complete and not compactoid after Proposition 1.10. Similarly, the rank-2 ring of integers of $F$ shares all these properties with $\mathcal{O}_{F}$.

Regarding duality, the fact that the map $\gamma: F \rightarrow F_{c}^{\prime}$ is an isomorphism of locally convex spaces does not change when we restrict scalars to $K$. Explicit nonzero linear forms $F \rightarrow K$ may be constructed by composing the maps $\pi_{i}: F \rightarrow \widetilde{K}$ as in (14) with $\operatorname{Tr} \widetilde{K} \mid K$.

Problem. It is relevant to decide whether the class of bounded sets of $F$ changes along with the change of vector space and locally convex structures associated to the choice of a different coefficient field.

\subsection{Mixed characteristic}

If char $F \neq \operatorname{char} \bar{F}$, then, as explained in $\Varangle 2$, there is a unique field embedding $\mathbb{Q}_{p} \hookrightarrow F$. If we denote the algebraic closure of $\mathbb{Q}_{p}$ in $F$ by $\widetilde{K}$, the field inclusion $K \hookrightarrow F$ may be factored into the following diagram of field embeddings

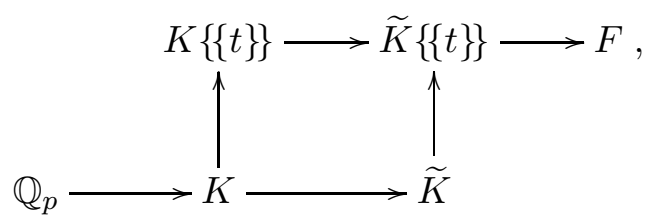

in which all horizontal arrows are finite extensions.

The inclusions $K \hookrightarrow K\{\{t\}\}$ and $\widetilde{K} \hookrightarrow \widetilde{K}\{\{t\}\}$ correspond to the situation we have been dealing with in the preceding sections of this work. Let $n=[F: \widetilde{K}\{\{t\}\}]$.

As $\widetilde{K}$-vector spaces, we have

$$
F \cong \widetilde{K}\{\{t\}\}^{n} .
$$

The higher topology on $F$ may be defined as the product topology on $n$ copies of the higher topology on $\widetilde{K}\{\{t\}\}$ [15, 1.3.2]. Furthermore, it does not depend on any choices of subfields $\widetilde{K}\{\{t\}\} \subset F[12, \S 1]$. Hence, since the product topology on a product of locally convex vector spaces is locally convex, the inclusion $\widetilde{K} \hookrightarrow F$ gives

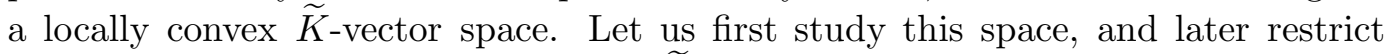
scalars along the finite extension $K \hookrightarrow \widetilde{K}$.

We may describe the family of open lattices or, equivalently, continuous seminorms, from the corresponding lattices or seminorms for $\widetilde{K}\{\{t\}\}$ and Proposition 1.1 ,

The situation for the ring of integers $\mathcal{O}_{F}$ is as follows. If we denote $\widetilde{\mathcal{O}}=\mathcal{O}_{\widetilde{K}}$, the inclusion $\widetilde{\mathcal{O}}\{\{t\}\} \hookrightarrow \mathcal{O}_{F}$ turns $\mathcal{O}_{F}$ into a rank- $n$ free $\widetilde{\mathcal{O}}\{\{t\}\}$-module. Therefore the subspace topology on $\mathcal{O}_{F} \subset F$ coincides with the product topology on $\mathcal{O}_{F} \cong \widetilde{\mathcal{O}}\{\{t\}\}^{n}$. From here, it is possible to show that $\mathcal{O}_{F}$ is a bounded and complete $\widetilde{\mathcal{O}}$-submodule of $F$ which is neither c-compact nor compactoid. It is however closed, but not open, and this proves that $F$ is not barrelled. The norm attached to the valuation $v_{F}$ provides a example of a seminorm which is bounded on bounded sets but not continuous, as its unit ball, $\mathcal{O}_{F}$, is not open. Hence $\widetilde{K} \hookrightarrow F$ is not bornological. 
From the self-duality of $\widetilde{K}\{\{t\}\}$, we obtain a chain of isomorphisms of locally convex $\widetilde{K}$-vector spaces

$$
F_{c}^{\prime} \cong\left(\widetilde{K}\{\{t\}\}^{n}\right)_{c}^{\prime} \cong\left(\widetilde{K}\{\{t\}\}_{c}^{\prime}\right)^{n} \cong \widetilde{K}\{\{t\}\}^{n} \cong F,
$$

which shows that $F$ is also self-dual. Explicit nonzero linear forms may be constructed in this case composing the trace map $\operatorname{Tr}_{F \mid \widetilde{K}\{\{t\}\}}$ with the maps $\pi_{i}: \widetilde{K}\{\{t\}\} \rightarrow$

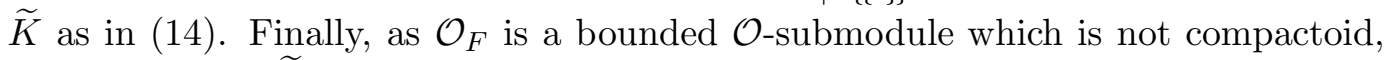
we deduce that $\widetilde{K} \hookrightarrow F$ is not nuclear.

In order to conclude our discussion, we need to verify that the properties of $K \hookrightarrow F$ agree with the ones of $\widetilde{K} \hookrightarrow F$. The discussion is very similar to what has already been discussed in 97.1 . In this case, the higher topology on $F$ is known not to depend on any choices [12, §1]. Similarly to what happens in the equal characteristic case, $\widetilde{\mathcal{O}}$-lattices are also $\mathcal{O}$-lattices after restriction of scalars and therefore the sets of open lattices and admissible seminorms for $\widetilde{K} \hookrightarrow F$ and $K \hookrightarrow F$ coincide. This also implies that the collections of bounded sets agree.

Suppose now that $B \subset F$ is a compactoid $\widetilde{\mathcal{O}}$-module. Given any open lattice $\Lambda \subset F$, there are $x_{1}, \ldots, x_{m} \in F$ such that $B \subset \Lambda+\widetilde{\mathcal{O}} x_{1}+\cdots+\widetilde{\mathcal{O}} x_{m}$. We have that $\widetilde{\mathcal{O}}$ is a free $\mathcal{O}$-module of rank $e=e(\widetilde{K} \mid K)$ and, in particular, there are $y_{1}, \ldots, y_{e} \in \widetilde{\mathcal{O}}$ such that $\widetilde{\mathcal{O}}=\mathcal{O} y_{1}+\cdots+\mathcal{O} y_{e}$. Hence, we have

$$
B \subseteq \Lambda+\sum_{i=1}^{m}\left(\sum_{j=1}^{e} \mathcal{O} y_{j} x_{i}\right)
$$

and therefore $B$ is a compactoid $\mathcal{O}$-submodule of $F$.

Reciprocally, if $B$ is an $\widetilde{\mathcal{O}}$-module which is a compactoid $\mathcal{O}$-submodule of $F$ after restriction of scalars, then for any open lattice $\Lambda$ there are finitely many $x_{1}, \ldots, x_{m} \in$ $F$ such that $B \subseteq \Lambda+\mathcal{O} x_{1}+\cdots+\mathcal{O} x_{m}$. But then we have inclusions

$$
B \subseteq \Lambda+\mathcal{O} x_{1}+\cdots+\mathcal{O} x_{m} \subseteq \Lambda+\widetilde{\mathcal{O}} x_{1}+\cdots+\widetilde{\mathcal{O}} x_{m}
$$

and, therefore, $B$ is also compactoid as an $\widetilde{\mathcal{O}}$-module.

It is for these reasons that $\mathcal{O}_{F}$ is an $\mathcal{O}$-lattice which is closed and complete but not open, bounded but not compactoid. Hence $K \hookrightarrow F$ is neither barrelled nor nuclear. Since any reflexive space is barrelled (Proposition 1.13), $F$ cannot be reflexive. The norm attached to the lattice $\mathcal{O}_{F}$ is bounded on bounded sets but not continuous since $\mathcal{O}_{F}$ is not open and, therefore, $K \hookrightarrow F$ is not bornological.

Finally, the isomorphisms of locally convex $\widetilde{K}$-vector spaces

$$
F_{c}^{\prime} \cong\left(\widetilde{K}\{\{t\}\}^{n}\right)_{c}^{\prime} \cong\left(\widetilde{K}\{\{t\}\}_{c}^{\prime}\right)^{n} \cong \widetilde{K}\{\{t\}\}^{n} \cong F
$$

turn into isomorphisms of locally convex $K$-vector spaces after restriction of scalars. Finally, we construct explicit non-zero linear forms on $F$ by, for example, taking

$$
\operatorname{Tr}_{\widetilde{K} \mid K} \circ \pi_{i} \circ \operatorname{Tr}_{F \mid \widetilde{K}\{\{t\}\}}: F \rightarrow \widetilde{K}\{\{t\}\} \rightarrow \widetilde{K} \rightarrow K, \quad i \in \mathbb{Z} .
$$




\section{A note on the archimedean case}

Let $\mathbb{K}=\mathbb{R}$ or $\mathbb{C}$. We will denote by $|\cdot|$ either the usual absolute value on $\mathbb{R}$, or the module on $\mathbb{C}$.

In this section we will consider the study of archimedean two-dimensional local fields. An archimedean two-dimensional local field is a complete discrete valuation field $F$ whose residue field is an archimedean (one-dimensional) local field. Hence, we have a non-canonical isomorphism $F \cong \mathbb{K}((t))$ for one of our two choices of $\mathbb{K}$. Once an inclusion of fields $\mathbb{K} \subset F$ has been fixed and $t$ has been chosen, a unique such isomorphism is determined.

The theory of locally convex vector spaces over $\mathbb{K}$ was developed much earlier than the analogous non-archimedean theory and is well explained in, for example, [13]. Let $V$ be a $\mathbb{K}$-vector space and $C \subseteq V$. The subset $C$ is said to be convex if for any $v, w \in C$, the segment

$$
\left\{\lambda v+\mu w ; \lambda, \mu \in \mathbb{R}_{\geq 0}, \lambda+\mu=1\right\}
$$

is contained in $C$. The subset $C$ is said to be absolutely convex if, moreover, we have $\lambda C \subseteq C$ for every $\lambda \in \mathbb{K}$ such that $|\lambda| \leq 1$.

We may associate a seminorm $p_{C}$ to any convex subset $C \subseteq V$ by the rule

$$
p_{C}: V \rightarrow \mathbb{R}, \quad x \mapsto \inf _{\rho>0, x \in \rho C} \rho .
$$

This seminorm satisfies the usual triangle inequality, but not the ultrametric inequality.

Definition 8.1. The $\mathbb{K}$-vector space $V$ is said to be locally convex if it is a topological vector space such that its topology admits a basis of neighbourhoods of zero given by convex sets.

It may be shown that if $V$ is locally convex its filter of neighbourhoods of zero also admits a basis formed by absolutely convex subsets [13, §18.1].

The higher topology on $\mathbb{K}((t))$ is defined following the procedure outlined in 33.1 . In this case, we consider the disks of $\mathbb{K}$ centered at zero and of rational radius; this defines a countable basis of convex neighbourhoods of zero for the euclidean topology on $\mathbb{K}$. Denote

$$
D_{\rho}=\{a \in \mathbb{K} ;|a|<\rho\}, \quad \rho \in \mathbb{Q}_{>0} \cup\{\infty\} .
$$

Given a sequence $\left(\rho_{i}\right)_{i \in \mathbb{Z}} \subset \mathbb{Q}_{>0} \cup\{\infty\}$ such that there is an index $i_{0}$ satisfying that $\rho_{i}=\infty$ for all $i \geq i_{0}$, consider the set

$$
\mathcal{U}=\sum_{i \in \mathbb{Z}} D_{\rho_{i}} t^{i} \subset F
$$

The sets of the form (17) form a basis of neighbourhoods of zero for the higher topology on $F$.

Proposition 8.2. The higher topology on $F$ is locally convex, in the sense of Definition 8.1. 
Proof. As the discs $D_{\rho_{i}}$ are convex, given two elements $x, y \in \mathcal{U}$, it is easy to check that the segment

$$
\left\{\lambda x+\mu y ; \lambda, \mu \in \mathbb{R}_{\geq 0} ; \lambda+\mu=1\right\}
$$

is contained in $\mathcal{U}$ by checking on each coefficient separately.

Thus, the basis of open neighbourhoods of zero described by the sets of the form (17) consists of convex sets, and hence the higher topology on $F$ is locally convex.

As we have done in the rest of cases, we may now describe the higher topology in terms of seminorms.

Proposition 8.3. Let $k \in \mathbb{Z}$. Given a sequence $\rho_{i} \in \mathbb{Q}_{>0} \cup\{\infty\}$ for every $i \leq k$, such that $\rho_{k}<\infty$, consider the seminorm

$$
\|\cdot\|: \mathbb{K}((t)) \rightarrow \mathbb{R}, \quad \sum_{i \geq i_{0}} x_{i} t^{i} \mapsto \max _{i \leq k} \frac{\left|x_{i}\right|}{\rho_{i}}
$$

having in mind the convention that $a / \infty=0$ for every $a \in \mathbb{R}_{\geq 0}$. The higher topology on $F$ is defined by the set of seminorms specified by (18).

Proof. We will show that the seminorm $\|\cdot\|$ defined by (18) is the gauge seminorm attached to the basic open neighbourhood of zero $\mathcal{U}$ given by (17).

Let $x=\sum_{i \geq i_{0}} x_{i} t^{i} \in F$ and $\rho>0$. If $k<i_{0}$, we may take $\rho=0$ and deduce that $q(x)=0$.

Otherwise, $x \in \rho \mathcal{U}$ if and only if $x_{i} \in \rho D_{\rho_{i}}$ for every $i_{0} \leq i \leq k$.

From this, we may deduce that $x \in \rho \mathcal{U}$ if and only if

$$
\frac{\left|x_{i}\right|}{\rho_{i}}<\rho \text { for every } i_{0} \leq i \leq k .
$$

Finally, the infimum value of $\rho$ satisfying (19) is precisely the maximum of the values $\left|x_{i}\right| / \rho_{i}$ for $i_{0} \leq i \leq k$.

We have described the higher topology on $\mathbb{K}((t))$ in a fashion that matches what has been done in the previous sections. However, this locally convex space often arises in functional analysis in the following way. We write

$$
\mathbb{K}((t))=\cup_{i \in \mathbb{N}} t^{-i} \cdot \mathbb{K} \llbracket t \rrbracket,
$$

Each component in the union is isomorphic to $\mathbb{K}^{\mathbb{N}}$, topologized using the product topology, and the limit acquires the strict inductive limit locally convex topology.

It is known that $\mathbb{K} \llbracket t \rrbracket$ is a Fréchet space, that is, complete and metrizable. As such, the two-dimensional local field $\mathbb{K}((t))$ is an LF-space and many of its properties may be deduced from the general theory of LF-spaces, see for example [13, §19]. In particular, $\mathbb{K}((t))$ is complete, bornological and nuclear. 


\section{A note on the characteristic $p$ case}

Let $k=\mathbb{F}_{q}$ be a finite field of characteristic $p$. In this section we will consider the two-dimensional local field $F=k((u))((t))$. It is a vector space both over the finite field $k$ and over the local field $k((u))$.

The higher topology on $F$ may be dealt with in two ways from a linear point of view. The first approach was started by Parshin [19], and it regards $F$ as a $k$-vector space. In this approach, $k$ is regarded as a discrete topological field and the tools used are those of linear topology, see [11, §1] for an account. Linear topology was first introduced by Lefschetz [14].

The work developed in the previous sections of this work may be applied and we may regard $F$ a locally convex $k((u))$-vector space. In this section we will explain that in this case we have obtained nothing new.

A topology on a $k$-vector space is said to be linear if the filter of neighbourhoods of zero admits a collection of linear subspaces as a basis. A linearly topological vector space $V$ is said to be linearly compact if any family $A_{i} \subset V, i \in I$ of closed affine subspaces such that $\bigcap_{i \in J} A_{i} \neq \emptyset$ for any finite set $J \subset I$, then $\bigcap_{i \in I} A_{i} \neq \emptyset$. Finally, a linearly topological vector space is locally linearly compact if it has a basis of neighbourhoods of zero formed by linearly compact open subspaces.

Let $\mathbf{V e c t}_{k}$ be the category of linearly topological $k$-vector spaces. Similarly, let $\operatorname{Vect}_{k((u))}$ be the category of locally convex $k((u))$-vector spaces.

Proposition 9.1. The rule

$$
\operatorname{Vect}_{k((u))} \rightarrow \operatorname{Vect}_{k},
$$

which restricts scalars on $k((u))$-vector spaces along the inclusion $k \hookrightarrow k((u))$ and preserves topologies and linear maps, is a functor.

Proof. Let $V$ be a locally convex $k((u))$-vector space, and let $\Lambda$ denote an open lattice. As the lattice $\Lambda$ is an $\mathcal{O}_{k((u))}$-module and we have the inclusion $k \hookrightarrow \mathcal{O}_{k(u))}=k \llbracket u \rrbracket$, it is also a $k$-vector space by restriction of scalars.

As the collection of open lattices $\Lambda$ is a basis for the filter of neighbourhoods of zero, $V$ is a linearly topological $k$-vector space and the first part of the proposition follows.

There is a strong analogy between the concepts of c-compactness for locally convex $k((u))$-vector spaces and linear compactness for linearly topological $k$-vector spaces; both definitions agree if in Proposition 1.7 we translate the words closed convex subspace by closed affine subspace.

However, it is not true in general that restriction of scalars on a c-compact $k((u))$-vector space yields a linearly compact $k$-vector space: $k((u))$, being spherically complete, is a c-compact $k((u))$-vector space $[21, \S 12]$ which is not a linearly compact $k$-vector space.

The lack of an embedding of a finite field into a characteristic zero two-dimensional local field makes the linear topological approach unavailable in that setting; the locally convex approach to these fields is therefore to be regarded as analogous to the linear approach in positive characteristic. Similarly, the language of locally convex spaces is to be regarded as one which unifies the approach to the zero characteristic and positive characteristic cases. 


\section{Future work}

We outline some directions which we consider interesting to explore in order to apply and extend the results in this work.

$\mathcal{O}$-linear locally convex approach to higher topology. In this work we have been able to deduce many properties about $K((t))$, either in an explicit or implicit way, from the fact that it is an LF-space, i.e.: an inductive limit of Fréchet spaces. This is not the case in mixed characteristics: the field $K\{\{t\}\}$ is not a direct limit of nice $K$-vector spaces. It is, however, a direct limit of $\mathcal{O}$-modules by construction.

The development of a theory of locally convex $\mathcal{O}$-modules, with topologies defined by seminorms, and the constructions arising within that theory, particularly those of initial and final locally convex topologies, would allow us to recover on one hand the results we have established for $K((t))$, and on the other hand they would let us describe $K\{\{t\}\}$ as a direct limit of perhaps nice $\mathcal{O}$-modules; this could be an extremely helpful contribution to the study of mixed characteristic two-dimensional local fields.

Generalization to higher local fields. If $F$ is a characteristic zero $n$-dimensional local field, then it is possible to exhibit a field embedding $K \hookrightarrow F$ and treat $F$ as a $K$-vector space. A higher topology on $F$ may be constructed inductively using the same procedures outlined at the beginning of 93 , see [15]. Therefore, it may be shown that these topologies define locally convex structures over $K$. Although the situation is slightly more complex, a systematic study of the functional theoretic properties of these locally convex spaces would be interesting to develop. The first steps in this direction have been taken in [2].

Study of $\mathcal{L}(F)$. As we have explained, the ring of continuous $K$-linear endomorphisms of a two-dimensional local field can be topologized and studied from a functional analytic point of view. It contains several relevant two-sided ideals defined by imposing certain finiteness conditions to endomorphisms. The most important of such ideals is the subspace of nuclear maps. Nuclear endomorphisms of a locally convex space play a distinguished role in the study of the properties of such space. In particular, the usual trace map on finite-rank operators extends by topological arguments to the subspace of nuclear endomorphisms. Establishing a characterization of nuclear endomorphisms of two-dimensional local fields is an affordable goal.

Multiplicative theory of two-dimensional local fields. Multiplication $\mu$ : $F \times F \rightarrow F$ on a two-dimensional field $F$ is not continuous as explained in $\$ 3.3$. It is a well-known fact that the map $\mu$ is sequentially continuous, and the sequential topological properties of higher topologies have been studied and applied successfully to higher class field theory [7] and to topologize sets of rational points of schemes over higher local fields [3].

However, for any $x \in F$, the linear maps

$$
\begin{aligned}
& \mu(x, \cdot): F \rightarrow F, \\
& \mu(\cdot, x): F \rightarrow F
\end{aligned}
$$


are continuous. This means that, in the terms of [21, §17], $\mu$ is a separately continuous bilinear map and therefore induces a continuous linear map of locally convex spaces

$$
\mu: F \otimes_{K, \iota} F \rightarrow F,
$$

where $F \otimes_{K, L} F$ stands for the tensor product $F \otimes_{K} F$ topologized using the inductive tensor product topology.

This suggests that besides the applications of the theory of semitopological rings to the study of arithmetic properties of higher local fields [23], we have the following new approach to the topic: a two-dimensional local field $F$ is a locally convex $K$ vector space endowed with a continuous linear map $\mu: F \otimes_{K, \iota} F \rightarrow F$ satisfying the usual axioms of multiplication.

After Proposition 4.9 and Corollary 5.12, another possible way to look at a twodimensional local field and deal with its multiplicative structure is as a bornological $K$-algebra, that is: $F$ is a $K$-algebra endowed with a bornology (that generated by bounded submodules or compactoid submodules) such that all $K$-algebra operations

$$
\begin{aligned}
& \sigma: F \times F \rightarrow F \quad \text { (addition), } \\
& \varepsilon: K \times F \rightarrow F \quad \text { (scalar multiplication), } \\
& \mu: F \times F \rightarrow F
\end{aligned}
$$

are bounded.

It is interesting to decide if the arithmetic properties of $F$ can be recovered from these contexts, and it would even more interesting to establish new connections between this functional analytic approach to higher topology and the arithmetic of $F$.

Functional analysis on adelic rings and modules over them. There are several two-dimensional adelic objects which admit a formulation as a restricted product of two-dimensional local fields and their rings of integers, which in our characteristic zero context were introduced by Beilinson [1] and Fesenko [5] (see [16, $\S 8]$ for a discussion of the topic). From what we have exhibited in this work, at least in dimension two, these adelic objects may be studied using the theory of locally convex spaces, archimedean or nonarchimedean.

Topological approach to higher measure and integration. The study of measure theory, integration and harmonic analysis on two-dimensional local fields is an interesting problem. A theory of measure and integration has been developed on two-dimensional local fields $F$ by lifting the Haar measure of the local field $\bar{F}$, 4 , [18. This theory relies heavily on the relation between $F$ and $\bar{F}$. The approach to measure and integration on $F$ using the functional theoretic tools arising from the relation between $F$ and $K$ could yield an alternative integration theory.

\section{References}

[1] A. A. Beĭlinson. Residues and adèles. Funktsional. Anal. i Prilozhen., 14(1):44$45,1980$. 
[2] A. Cámara. Locally convex structures on higher local fields. 15p. Submitted. arXiv:1210.8068,

[3] A. Cámara. Topology on rational points over higher local fields. 23p. Awaiting for publication at Journal of Algebra and Number Theory. arXiv:1106.0191v1.

[4] I. Fesenko. Analysis on arithmetic schemes. I. Doc. Math., (Extra Vol.):261-284 (electronic), 2003. Kazuya Kato's fiftieth birthday.

[5] I. Fesenko. Analysis on arithmetic schemes. II. J. K-Theory, 5(3):437-557, 2010.

[6] I. Fesenko and M. Kurihara, editors. Invitation to higher local fields, volume 3 of Geometry $\&$ Topology Monographs. Geometry \& Topology Publications, Coventry, 2000. Papers from the conference held in Münster, August 29-September 5,1999 .

[7] I. B. Fesenko. Sequential topologies and quotients of Milnor $K$-groups of higher local fields. Algebra i Analiz, 13(3):198-221, 2001.

[8] I. B. Fesenko and S. V. Vostokov. Local fields and their extensions, volume 121 of Translations of Mathematical Monographs. American Mathematical Society, Providence, RI, second edition, 2002. With a foreword by I. R. Shafarevich.

[9] H. Hogbe-Nlend. Théorie des bornologies et applications. Lecture Notes in Mathematics, Vol. 213. Springer-Verlag, Berlin, 1971.

[10] H. Hogbe-Nlend. Bornologies and functional analysis. North-Holland Publishing Co., Amsterdam, 1977. Introductory course on the theory of duality topology-bornology and its use in functional analysis, Translated from the French by V. B. Moscatelli, North-Holland Mathematics Studies, Vol. 26, Notas de Matemática, No. 62. [Notes on Mathematics, No. 62].

[11] M. Kapranov. Semiinfinite symmetric powers. arXiv:math/0107089v1.

[12] K. Kato. Existence theorem for higher local fields. In Invitation to higher local fields (Münster, 1999), volume 3 of Geom. Topol. Monogr., pages 165-195. Geom. Topol. Publ., Coventry, 2000.

[13] G. Köthe. Topological vector spaces. I. Translated from the German by D. J. H. Garling. Die Grundlehren der mathematischen Wissenschaften, Band 159. Springer-Verlag New York Inc., New York, 1969.

[14] S. Lefschetz. Algebraic Topology. American Mathematical Society Colloquium Publications, v. 27. American Mathematical Society, New York, 1942.

[15] A. I. Madunts and I. B. Zhukov. Multidimensional complete fields: topology and other basic constructions. In Proceedings of the St. Petersburg Mathematical Society, Vol. III, volume 166 of Amer. Math. Soc. Transl. Ser. 2, pages 1-34, Providence, RI, 1995. Amer. Math. Soc.

[16] M. Morrow. An introduction to higher local fields. Available at http://math.uchicago.edu/ mmorrow/Morrow\%20Intro\%20to\%20HLF.pdf. 
[17] M. Morrow. An explicit approach to residues on and dualizing sheaves of arithmetic surfaces. New York J. Math., 16:575-627, 2010.

[18] M. Morrow. Integration on valuation fields over local fields. Tokyo J. Math., $33(1): 235-281,2010$.

[19] A. N. Parshin. Local class field theory. Trudy Mat. Inst. Steklov., 165:143-170, 1984. Algebraic geometry and its applications.

[20] C. Perez-Garcia and W. H. Schikhof. Locally convex spaces over nonArchimedean valued fields, volume 119 of Cambridge Studies in Advanced Mathematics. Cambridge University Press, Cambridge, 2010.

[21] P. Schneider. Nonarchimedean functional analysis. Springer Monographs in Mathematics. Springer-Verlag, Berlin, 2002.

[22] J. T. Tate. Fourier analysis in number fields, and Hecke's zeta-functions. In Algebraic Number Theory (Proc. Instructional Conf., Brighton, 1965), pages 305-347. Thompson, Washington, D.C., 1967.

[23] A. Yekutieli. An explicit construction of the Grothendieck residue complex. Astérisque, (208):127, 1992. With an appendix by Pramathanath Sastry.

\section{Alberto Cámara}

School of Mathematical Sciences

University of Nottingham University Park Nottingham

NG7 2RD

United Kingdom

http://www.maths.nottingham.ac.uk/personal/pmxac

pmxac@nottingham.ac.uk 